\title{
Pulsed sextupole injection for Sweden's new light source MAX IV
}

\author{
S. C. Leemann* \\ MAX-lab, Lund University, S-22363 Lund, Sweden \\ (Received 21 November 2011; published 8 May 2012)
}

\begin{abstract}
The MAX IV facility presently under construction will include two storage rings for the production of synchrotron radiation. Both rings will be operated at a constant $500 \mathrm{~mA}$ of stored current with top-up shots supplied by the MAX IV linac acting as a full-energy injector. Until recently, injection into both storage rings was designed using a conventional approach: a closed four-kicker injection bump brings the stored beam to the septum blade where the injected bunches are captured in a single turn. This method, although commonly found in third generation light sources, has significant disadvantages. Therefore, an alternative injection into the storage rings using pulsed multipoles has been investigated. This type of injection does not require an injection bump and has the potential to make top-up injection fully transparent to users. Design studies have been successfully completed and as a consequence it has been decided to replace the originally foreseen conventional injection scheme with pulsed multipole injection in both MAX IV storage rings. This paper reports on these studies, presents pulsed sextupole injection schemes, and summarizes requirements for the pulsers, sextupole magnets, and vacuum chambers.
\end{abstract}

DOI: 10.1103/PhysRevSTAB.15.050705

PACS numbers: 29.20.db, 41.60.Ap, 29.27.Ac, 07.55.Db

\section{INTRODUCTION}

The MAX IV facility presently under construction $[1,2]$ will include two storage rings for the production of synchrotron radiation: the $3 \mathrm{GeV}$ storage ring [3] will house insertion devices for the production of $\mathrm{x}$ rays while the $1.5 \mathrm{GeV}$ storage ring [4] will serve UV and IR users. Both rings will be operated at a constant $500 \mathrm{~mA}$ of stored current with top-up shots supplied by the $3.5 \mathrm{GeV}$ MAX IV linac acting as a full-energy injector [5]. The linac will deliver low-emittance, low-energy spread bunches at up to $10 \mathrm{~Hz}$ to either ring via the two transfer lines.

The following sections will briefly summarize the expected linac injector performance and present the transfer line layout and top-up requirements for the storage rings. Section II will present the originally foreseen conventional injection scheme using a local four-kicker injection bump and point out the limitations and drawbacks of such a solution. Section III will then introduce the pulsed multipole injection as an alternative concept and describe advantages over the conventional scheme. In Secs. IV and V the new injection scheme for the MAX IV storage rings using a pulsed sextupole magnet is presented along with tracking results to demonstrate injection efficiency. Section VI reviews magnet, pulser, and vacuum chamber design specifications. Considerations regarding tolerances, alignment, and commissioning of the pulsed sextupole

\footnotetext{
*simon.leemann@maxlab.lu.se
}

Published by the American Physical Society under the terms of the Creative Commons Attribution 3.0 License. Further distribution of this work must maintain attribution to the author(s) and the published article's title, journal citation, and DOI. magnet injection system are also presented. Finally, Sec. VII offers a summary as well as an outlook on further studies.

\section{A. Linac injector}

During regular user operation the MAX IV linac delivers individual $3 \mathrm{GHz}$ bunches to the MAX IV Short Pulse Facility (SPF) [6] at $100 \mathrm{~Hz}$. These bunches originate from a photocathode rf gun and pass two stages of compression. This cycle is interrupted briefly for top-up injection shots to the two MAX IV storage rings. For top-up shots, bunches are extracted from a thermionic rf gun with chopper [7], accelerated by the linac without compression, and kicked into one of two transfer lines to the storage rings at a maximum repetition rate of $10 \mathrm{~Hz}$. Each top-up injection shot consists of a bunch train of three $3 \mathrm{GHz}$ linac shots $(3 \times 100 \mathrm{pC})$ that are injected into one common $100 \mathrm{MHz}$ bucket in either storage ring. Such bunches are expected to have a normalized emittance $\varepsilon_{n}=$ $10 \mathrm{~mm} \mathrm{mrad}$ and energy spread $\sigma_{\delta}=0.1 \%$. Each of the bunches in the train has a length of 5 ps (FWHM) at extraction which should limit peak current in the transfer lines to roughly $20 \mathrm{~A}$. Selection of a storage ring bucket to inject into is performed by the timing system. In combination with a fast photodiode at a diagnostic beam line, this system should allow operation of a filling pattern feedback.

\section{B. Top-up injection requirements}

Obviously, the exact choice of top-up deadband (which then dictates the filling pattern granularity) will determine the top-up injection rate. With the overall storage ring lifetimes estimated to be at least $10 \mathrm{~h}$ [3], top-up injection can be expected to occur as often as once every $12 \mathrm{~s}$ (one 
$300 \mathrm{pC}$ shot corresponding to a top-up deadband of $0.19 \%$ and $0.03 \%$ in the 1.5 and $3 \mathrm{GeV}$ storage ring, respectively) or as rarely as once every $6 \mathrm{~min}$ (for a $1 \%$ deadband corresponding to $5 \mathrm{~mA}$ in both rings). The storage rings can be operated in both scenarios: infrequent top-up injections with several shots or frequent injection of individual shots. The ultimate decision can be left to the user community, but regardless of the choice, a top-up injection which is as transparent and efficient as possible is highly desirable.

\section{Linac extraction and transfer lines}

In the linac there are two extraction points (EPs) to the two storage ring transfer lines: one at $1.5 \mathrm{GeV}$ and one at $3 \mathrm{GeV}$. Figure 1 shows an overview of the MAX IV facility including the vertical transfer lines linking the underground linac with the storage rings above ground.

Once a top-up shot is required, a bunch train from the thermionic gun (three $3 \mathrm{GHz}$ bunches) is accelerated and the linac extraction kickers upstream of the appropriate EP are triggered. This moves the bunch train into the extraction channel of the vertical DC septum $\left(10^{\circ}\right.$ and $5^{\circ}$ in the 1.5 and $3 \mathrm{GeV}$ storage ring, respectively). After the bunch train exits the septum it passes a vertical bending magnet $\left(17^{\circ}\right.$ and $16.5^{\circ}$, respectively) which bends the train into the straight section of the transfer line where the bunches pass the linac tunnel ceiling and enter the storage ring tunnel through the hall floor. In order to reduce complexity, the transfer lines are mirror symmetric around their centers. Three quadrupole families (each consisting of a pair of quadrupoles) are used to reproduce the optics of the EP in the linac at the storage ring injection point (end of injection

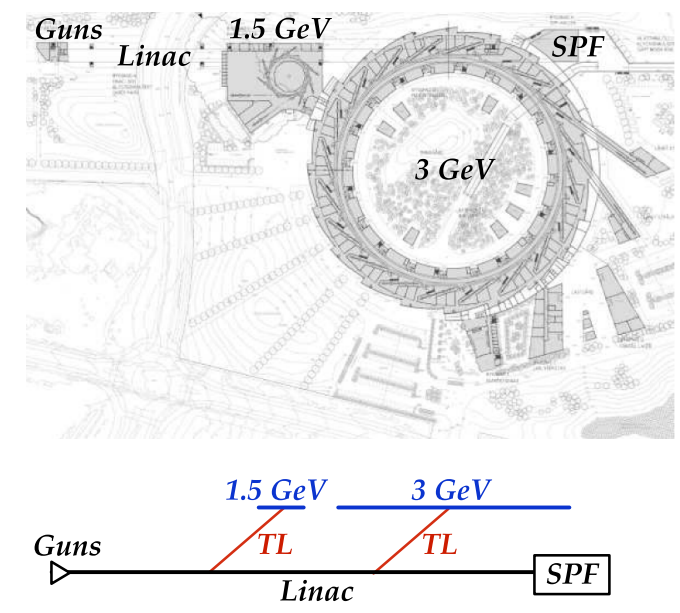

FIG. 1. Top: Layout of the MAX IV facility as seen from above. The guns, linac, and short-pulse facility (SPF) are underground. The 1.5 and $3 \mathrm{GeV}$ storage rings are above ground. Bottom: Schematic of the injection system for the MAX IV storage rings as seen from the side. Two vertical transfer lines (TL) connect the linac extraction points with the storage ring injection points. septum) and to maintain achromaticity (cf. Figs. 2 and 3). The linac optics however, are not optimized for injection into the storage rings. Linac optics are matched to the bunch compressors. It is the large storage ring acceptance combined with the low-emittance and low-energy spread bunches from the linac that render high capture efficiency (cf. Secs. IVA and VA). This is an important advantage since the linac optics do not have to be readjusted for topup injection shots.

At the end of the transfer lines, a vertical bending magnet and Lambertson septum (a modification of the design presented in $[8,9]$ ) bend the injected bunch train into the horizontal plane so that it coasts alongside the stored beam. The septum blade is at $-10 \mathrm{~mm}[-13.5 \mathrm{~mm}]$ from the position of the unperturbed stored beam in the $3 \mathrm{GeV}$ storage ring [1.5 GeV storage ring] and is $2.5 \mathrm{~mm}$ thick in both rings. The horizontal acceptance of the storage ring is $11 \mathrm{~mm}$ mrad [32 $\mathrm{mm} \mathrm{mrad}]$. In this paper, the magnetic end of this septum shall be referred to as the injection point (IP). After this point the injection scheme of the storage ring needs to kick the injected bunches into the storage ring acceptance where they proceed to damp down. This shall be discussed in more detail in the rest of this paper.

\section{CONVENTIONAL INJECTION AND ITS LIMITATIONS}

The original injection scheme for the MAX IV storage rings was designed with a conventional local four-kicker injection bump that would bring the stored beam to $-8 \mathrm{~mm}[-10 \mathrm{~mm}]$, that is, within $5.5 \mathrm{~mm}[7 \mathrm{~mm}]$ of the injected bunch. After the injection bump, the injected bunch is within the acceptance of the storage ring and proceeds to damp down while it oscillates around the stored beam (cf. Fig. 4). With a transverse damping time of roughly $15 \mathrm{~ms}$ [6 ms], there is ample time for the injected bunch to damp down until the next shot is injected. Because of the horizontal tune of 42.20 [11.22], the injection bump needs to be ramped down within less than 4-5 revolution periods, i.e. $8.8 \mu \mathrm{s}$ [1.5 $\mu \mathrm{s}]$.

Since continuous top-up injection is foreseen, an important criterium for the choice of injection scheme is that topup shots should be as transparent to users as possible. Stored beam residual motion during top-up injection must be limited to within beam position stability criteria: $5 \mu \mathrm{m}[18 \mu \mathrm{m}]$ in the horizontal plane and $200 \mathrm{~nm}[1 \mu \mathrm{m}]$ in the vertical plane at the ID [1]. Especially the $200 \mathrm{~nm}$ vertical tolerance is difficult to achieve. In light of this aspect, the conventional four-kicker injection bump scheme has several disadvantages.

(i) Four dipole kickers and their pulsers need to be perfectly matched, synchronized, and aligned so the bump reaches its design amplitude and is properly closed (this places high demands on chamber coating homogeneity, pulsers, and cables). 


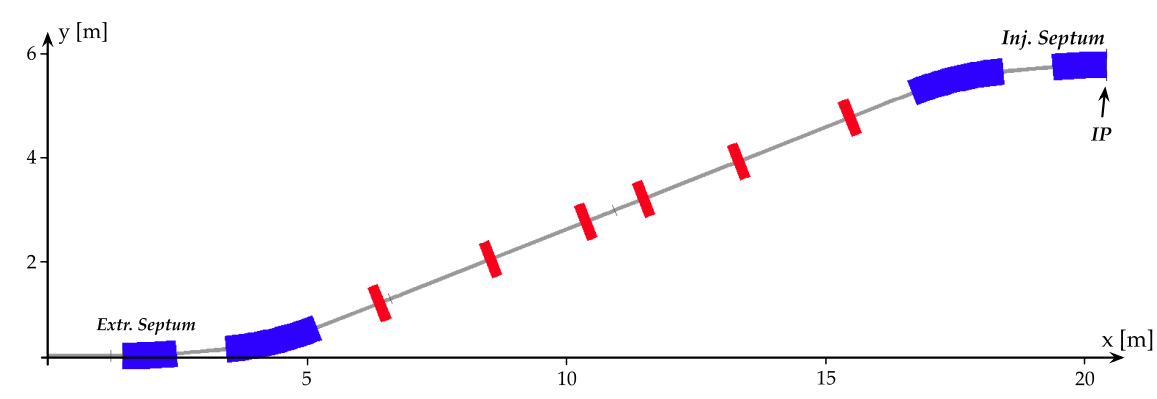

FIG. 2. Layout of the MAX IV $3 \mathrm{GeV}$ transfer line (analogous to the $1.5 \mathrm{GeV}$ transfer line). The linac extraction septum and storage ring injection septum as well as the two $16.5^{\circ}$ vertical dipoles are indicated in blue. Six quadrupoles installed symmetrically around the transfer line center are shown in red. The vertical displacement between injection and extraction is $5.6 \mathrm{~m}$.

(ii) If the local injection bump is not fully closed, a coherent betatron oscillation of the stored beam is excited. This leads to fluctuations of the electron beam position and intensity in the ID thus degrading the photon beam at the experiments.

(iii) Much space is required to house four strong dipole kickers and the septum. If more space is required than available in the injection straight (the MAX IV rings do not house extra-long straights for injection), injection elements may take up space otherwise reserved for IDs.

(iv) If sextupoles and/or octupoles are contained within the injection bump, the bump is only perfectly closed for one energy and one amplitude. The bump cannot be properly closed for particles with finite energy spread or if the bump cannot be opened and closed instantaneously. This remains an issue regardless of how well aligned and matched the dipole kickers are.

The first two points underline the complexity introduced by a four-kicker injection bump. Although this injection scheme is common in 3rd-generation light sources, operational experience shows that, despite vigorous correction and optimization efforts, top-up injection with a fourkicker injection bump cannot be made entirely transparent

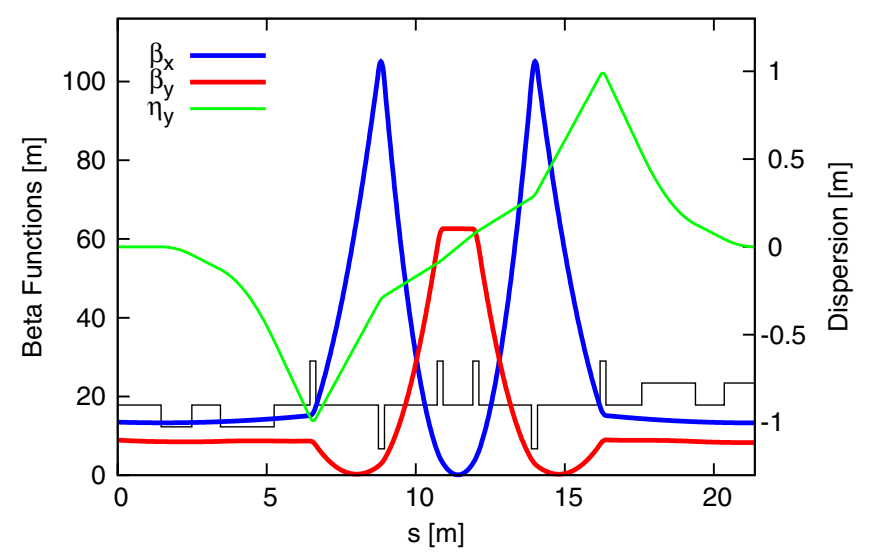

FIG. 3. Optics of the MAX IV $3 \mathrm{GeV}$ transfer line (analogous to the $1.5 \mathrm{GeV}$ transfer line). Data is shown starting upstream of the extraction septum and ending at the end of the storage ring injection septum (IP).
[10]. Furthermore, as stability criteria become tougher in newer storage ring designs, this method of injection becomes less favorable. Therefore, several labs have started investigating alternative injection schemes [11-15].

For the storage rings in the MAX IV facility, especially the last two points present a problem. In the $3 \mathrm{GeV}$ storage ring, strong sextupoles and octupoles are used to optimize chromatic and amplitude-dependent tune shifts [16]. As a consequence, nonlinear behavior for particles at large amplitudes (such as the bumped stored beam and the injected bunch) is very pronounced. Since the injection bump spans the injection straight as well as the upstream and downstream short straights (cf. Fig. 4), sextupoles, octupoles, and dispersion are all contained within the bump and it becomes impossible to perfectly close the bump for all particle energies in the stored beam. Additionally, while the bump is opened and closed, bunches sample reduced bump amplitudes. Because of the nonlinearity these reduced-amplitude bumps are not properly closed. Figures 5 and 6 show examples using the original fourkicker bump injection scheme in the $3 \mathrm{GeV}$ storage ring: the bump can only be closed properly for one energy and amplitude.

In the $1.5 \mathrm{GeV}$ storage ring there is only a single $3.5 \mathrm{~m}$ long straight between each of the 12 achromats. Because of the space required for kickers, septum, and transfer line elements, the injection bump must be opened in the straight upstream of the injection straight and closed downstream of it. Therefore, the injection bump spans at least two achromats. Both contain strong sextupoles and significant dispersion which again prevents perfect closure for all stored beam particles and all bump amplitudes. In addition, the length available for installation of insertion devices in the upstream and downstream straights is limited by two of the four injection kickers.

Therefore, it was decided to investigate alternative injection schemes for the storage rings in the MAX IV facility. A promising candidate is pulsed multipole injection (PMI) where a single multipole magnet is used to capture injected bunches without perturbing the stored beam. The next section will give a short introduction to PMI and summarize its advantages over conventional 

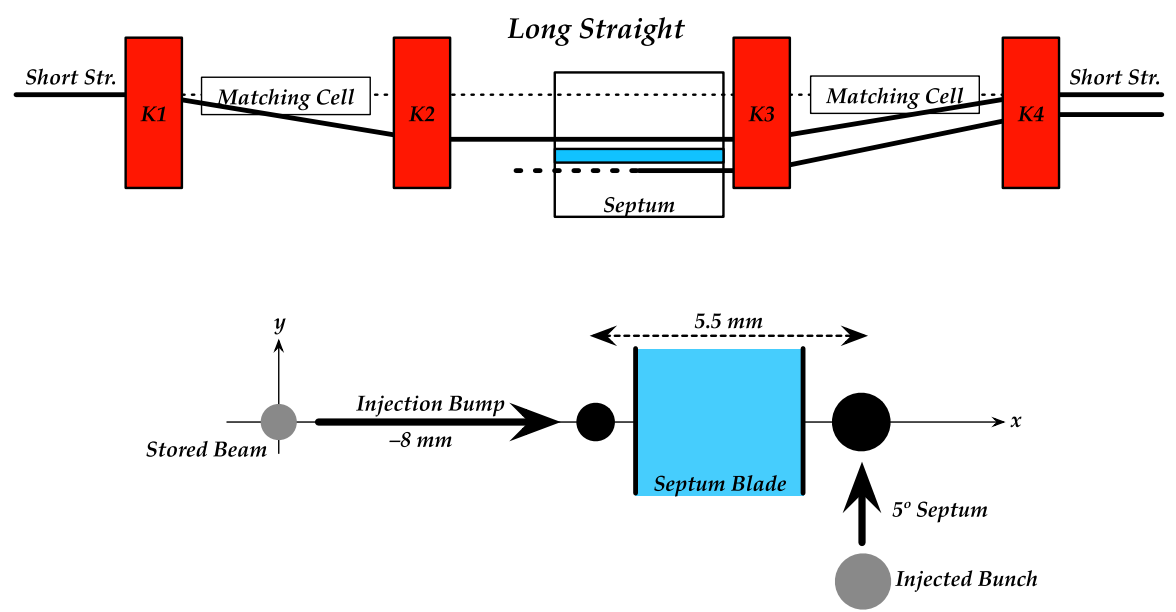

FIG. 4. Schematic of the conventional four-kicker bump injection scheme in the MAX IV $3 \mathrm{GeV}$ storage ring. Injection is displayed as seen from above (top) and in the transverse plane (bottom).
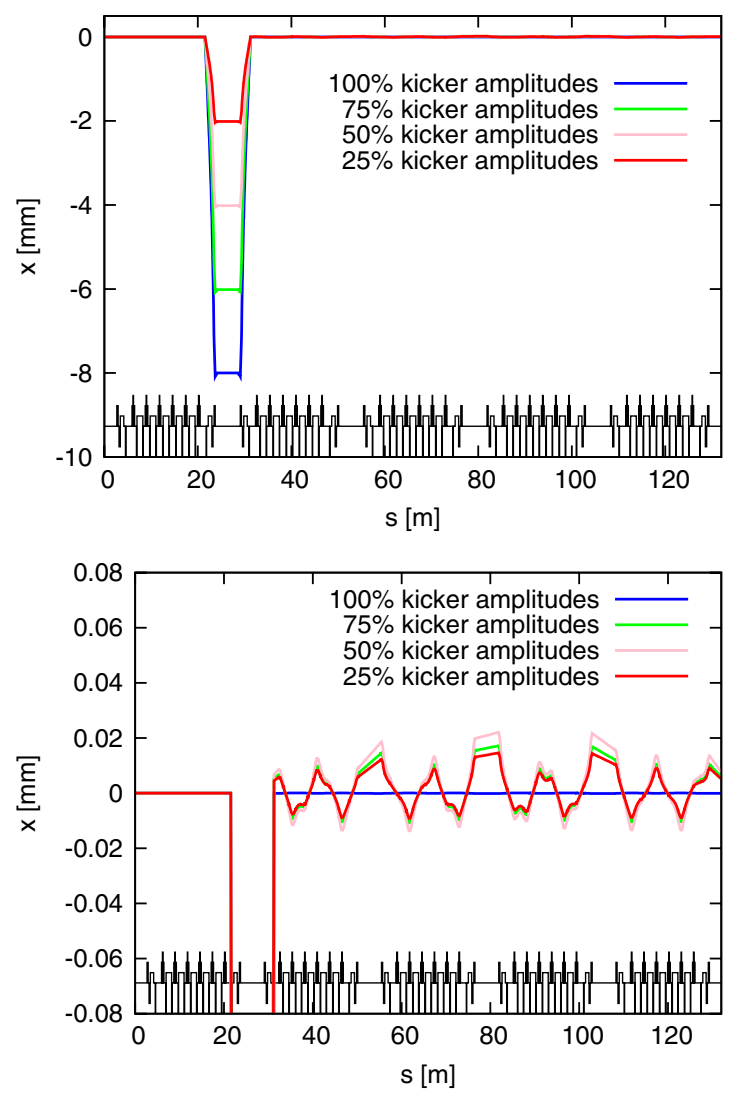

FIG. 5. While opening and closing the bump the kicker strength is ramped. The bump can however only be perfectly closed at one amplitude. At other amplitudes, i.e., during the ramp, residual betatron oscillations are excited. The bottom plot is an enlarged view of the top plot showing residual betatron oscillations downstream of the injection bump. Residual amplitudes of up to $22 \mu \mathrm{m}$ are observed at $50 \%$ kicker amplitude.
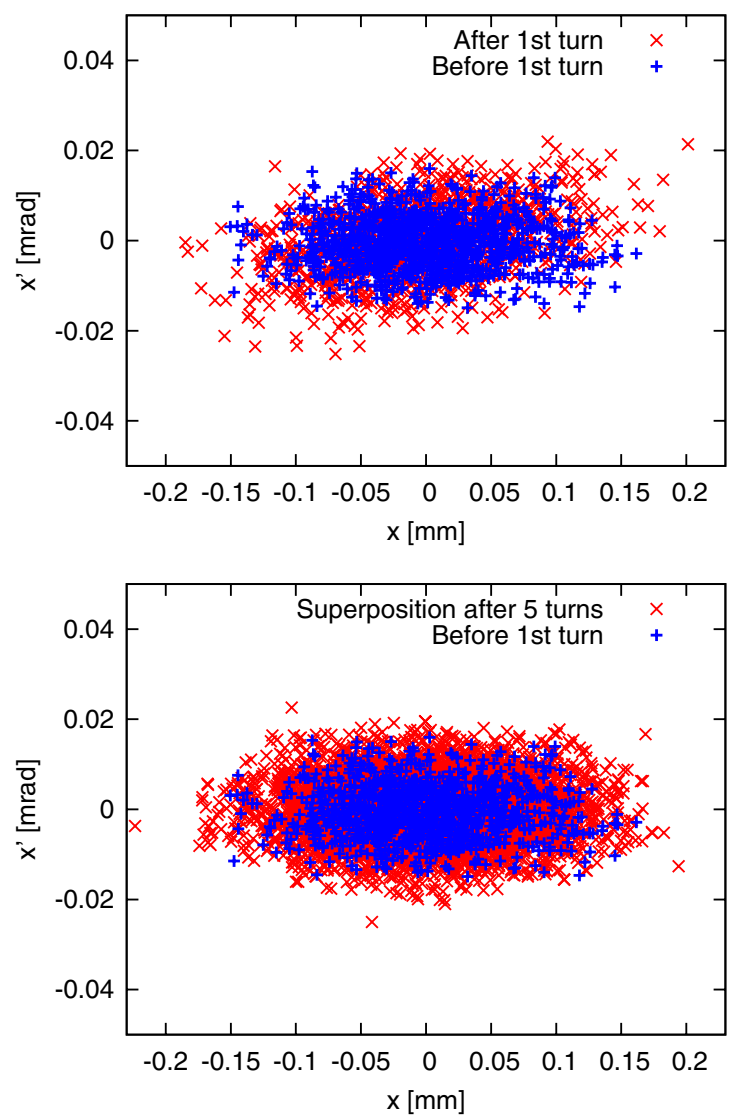

FIG. 6. Nonperfect closure of a conventional four-kicker injection bump in the MAX IV $3 \mathrm{GeV}$ ring. Top: Effect of bump on stored beam after one turn with kickers at full amplitude (closed bump). The apparent emittance blowup is caused by the energy spread in the bunch-the bump is not closed for all particles. Bottom: Effect of bump on stored beam with kickers at $50 \%$ amplitude (not properly closed bump). The apparent emittance blowup is caused by the residual betatron oscillation of the stored beam. 
injection with a four-kicker bump. The following sections then present an injection scheme for the MAX IV storage rings using a pulsed sextupole magnet.

\section{PULSED MULTIPOLE INJECTION}

In PMI capture is achieved without bumping the orbit of the stored beam. Instead, the injected bunch is kicked into the storage ring acceptance by a pulsed multipole magnet (cf. Fig. 7). Since the stored beam passes the multipole magnet through the magnetic center, it sees approximately zero field. The only synchronization required is between the pulser and the passage of the injected bunch. Alignment also becomes easier than in conventional fourkicker bump injection: the stored beam has to pass the magnetic center of only a single pulsed magnet. This can be achieved either by the orbit correction system or by beam-based alignment of the pulsed magnet. The pulse can last up to several revolution periods depending on the fractional tune. In most cases the exact pulse shape is not crucial as long as fall time is sufficiently fast. Since besides the septum, only a single magnet is required for capture, PMI requires substantially less space than conventional four-kicker bump injection.

In the most basic setup, a pulsed quadrupole magnet (PQM) can be used for this purpose. The stored beam passes the center of the PQM where the magnetic field is approximately zero while the injected bunch passes the $\mathrm{PQM}$ off center therefore receiving a dipole kick proportional to the offset from the magnetic center. The PQM injection scheme has been successfully designed and commissioned at the Photon Factory Advanced Ring (PF-AR) at KEK [11]. But PMI is not restricted to quadrupoles. In fact, there are advantages to using higher-order multipole magnets, for example a pulsed sextupole magnet (PSM) [15]. Around the magnet center, the PSM field is symmetric and flat: for a specific amplitude of the injected bunch at the PSM, $x_{\mathrm{pm}}$, the residual kick to a particle within the stored beam (at a small amplitude $x$ ) is reduced by a factor $x / x_{\mathrm{pm}}$ compared to the residual kick from a PQM. With typical amplitudes at injection on the order of $5 \mathrm{~mm}$ and typical horizontal beam sizes on the order of $50 \mu \mathrm{m}$, this ratio is on the order of $10^{2}$ and it becomes evident that a PSM perturbs the stored beam much less than a PQM.

This can be nicely demonstrated by tracking the stored beam through the PQM and PSM and comparing the effect of any residual kick received by the stored beam. Results of such a tracking study for the MAX IV $3 \mathrm{GeV}$ storage ring are shown in Fig. 8 where in each case the pulsed magnet was set to apply a -1.17 mrad kick to the injected beam during one turn. The pulsed magnets are assumed to have ideal field distribution and be perfectly aligned to the stored beam. The plots show the stored beam before and after the PQM/PSM has been pulsed. The stored beam horizontal emittance is $\varepsilon_{x}=0.326 \mathrm{~nm}$ rad which gives a horizontal $\mathrm{rms}$ beam size of $\sigma_{x}=55 \mu \mathrm{m}$ at the location shown. The effect of the PSM on the stored beam is hardly recognizable while the PQM shows significant perturbation of the stored beam.
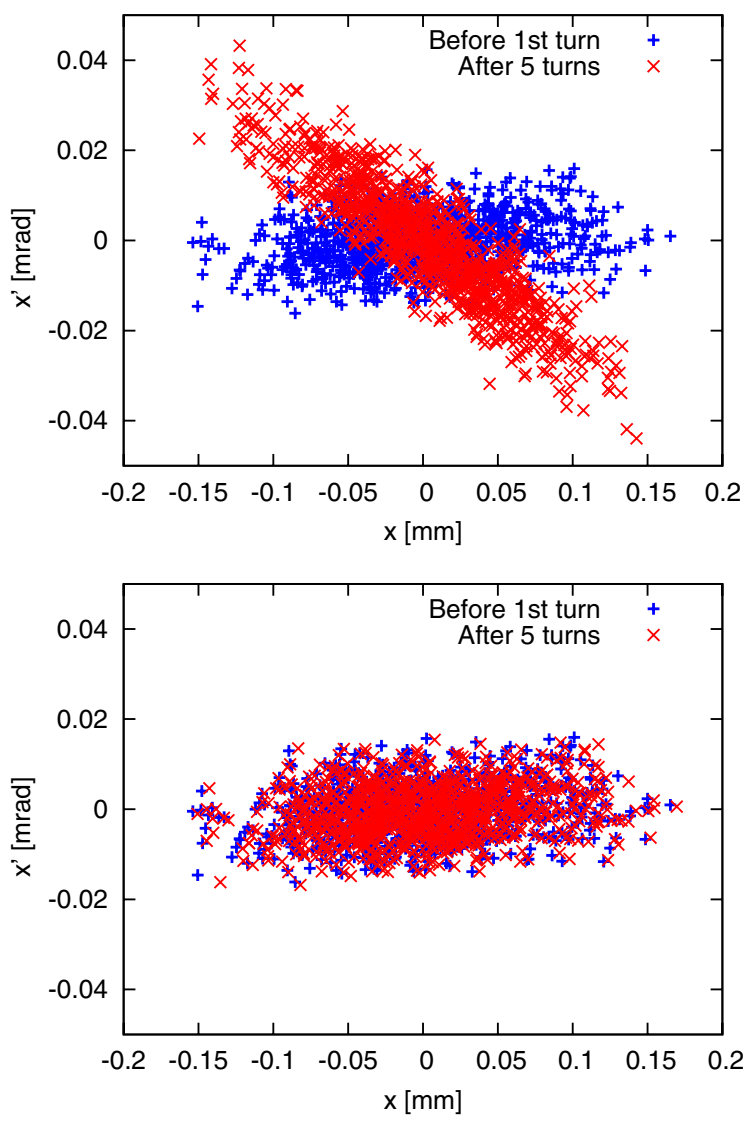

FIG. 8. DIMAD tracking results at the MAX IV $3 \mathrm{GeV}$ storage ring IP showing the effect of the pulsed magnet (single-turn injection mode) on the stored beam $\left(\varepsilon_{x}=0.326 \mathrm{~nm} \mathrm{rad}, \sigma_{\delta}=\right.$ $7.7 \times 10^{-4}, n=1000$, Gaussian distribution with cutoff at $3 \sigma$ ). The PQM (top) and the PSM (bottom) were both set to apply -1.17 mrad kick to the injected beam.

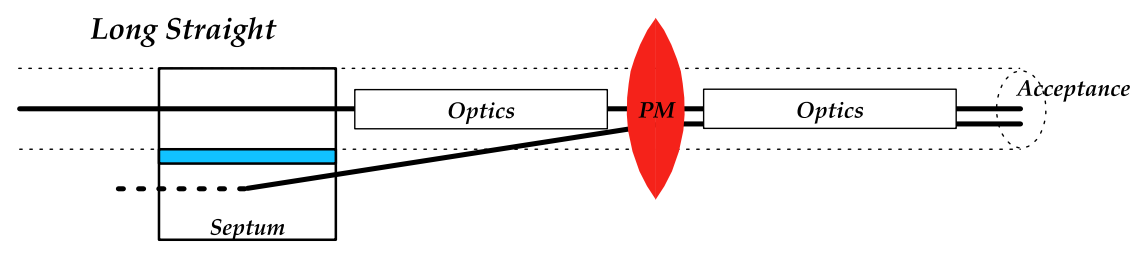

FIG. 7. Schematic of pulsed multipole injection in the MAX IV $3 \mathrm{GeV}$ storage ring. 
In fact, since the ideal magnet for PMI requires a large field component at $x_{\mathrm{pm}}$ and zero field elsewhere, even higher-order multipoles or irregular multipole magnets can be considered [10,17]. Ideally the transverse field distribution of such a magnet has a zero crossing so that the magnet can always be aligned in such a way that the stored beam passes the magnet at a zero-field location. However, if the transverse field distribution varies very strongly around the ideal position of the injected beam, $x_{\mathrm{pm}}$, injection becomes more sensitive to optics changes, most notably changes of the nonlinear optics. This leads to an obvious trade-off between desired flexibility and magnet design tailoring.

\section{Pulsed sextupole injection}

For the MAX IV facility, the focus was initially put on PSM injection since feasibility had been demonstrated and it is regarded as a reasonable compromise between expected performance and required development effort. Injection with a PSM was first implemented at the photon factory (PF) at KEK [15] and experience with PSM top-up injection has been very positive with electron and photon beam stability during injection significantly below previously observed levels $[18,19]$. The MAX IV storage rings will be the first storage ring light sources designed to use PMI from the start. A conventional four-kicker bump injection will not be implemented at all.

Starting with the coordinates of the injected bunch at the injection point $x_{\mathrm{inj}}$ and taking into account the storage ring's horizontal acceptance $A_{x}$ (since capture occurs in the horizontal plane this is the plane of interest here), an optimum location for a PSM can be derived. As has been shown in [15], the ideal location of the pulsed magnet is given by the injection invariant $A_{\text {inj }}$ and reduced invariant $A_{\text {red }}$ alone. The reduced invariant must obviously lie within the storage ring's acceptance: $A_{\text {red }}<A_{x}$. If $\phi_{\mathrm{pm}}$ is the phase advance between the symmetry point (usually close to the septum) and the pulsed magnet, the minimum required kick is achieved where

$$
\cos \phi_{\mathrm{pm}}= \pm \frac{A_{\mathrm{red}}}{A_{\mathrm{inj}}}
$$

and the required kick $\theta_{\mathrm{pm}}$ is then given by

$$
\theta_{\mathrm{pm}}=\frac{A_{\text {inj }}}{\sqrt{\beta_{\mathrm{pm}}}}\left|\sin \phi_{\mathrm{pm}}\right| \text {. }
$$

Since the pulsed magnet only applies a kick, the position of the injected bunch in the pulsed magnet $x_{\mathrm{pm}}$ must obviously already lie within the boundary set by the reduced invariant

$$
\left|\frac{x_{\mathrm{pm}}}{\sqrt{\beta_{\mathrm{pm}}}}\right|<A_{\text {red }}
$$

So, in principle, one could define a desired reduced invariant and from this derive the ideal position of the pulsed magnet and the required kick strength. Of course, ideal placement cannot necessarily be achieved because the lattice might not leave space for the pulsed magnet at that location. In practice, one usually tries to find an available drift space sufficiently close to the minimum position in order to keep the required kick feasible.

This picture is however too simple because of the underlying assumption that betatron motion is linear. In modern ring-based light sources like the MAX IV storage rings, there are usually strong sextupoles and/or octupole magnets that induce considerable nonlinearity in betatron motion, especially at the large amplitudes of injected bunches (cf. Fig. 9). Therefore, the actual pulsed magnet solution has to be derived from tracking. The approach is however similar: define a desired reduced invariant and from this derive candidate locations, i.e., locations where the amplitude of the injected bunch lies within the reduced invariant. A suitable location for the pulsed magnet is then an available drift space with nonzero divergence of the injected bunch $x_{\mathrm{pm}}^{\prime}$ where the amplitude of the injected bunch is not far below the maximum amplitude derived from the reduced invariant. This reduces the required pulsed sextupole strength $\left(b_{3} L\right)$ since
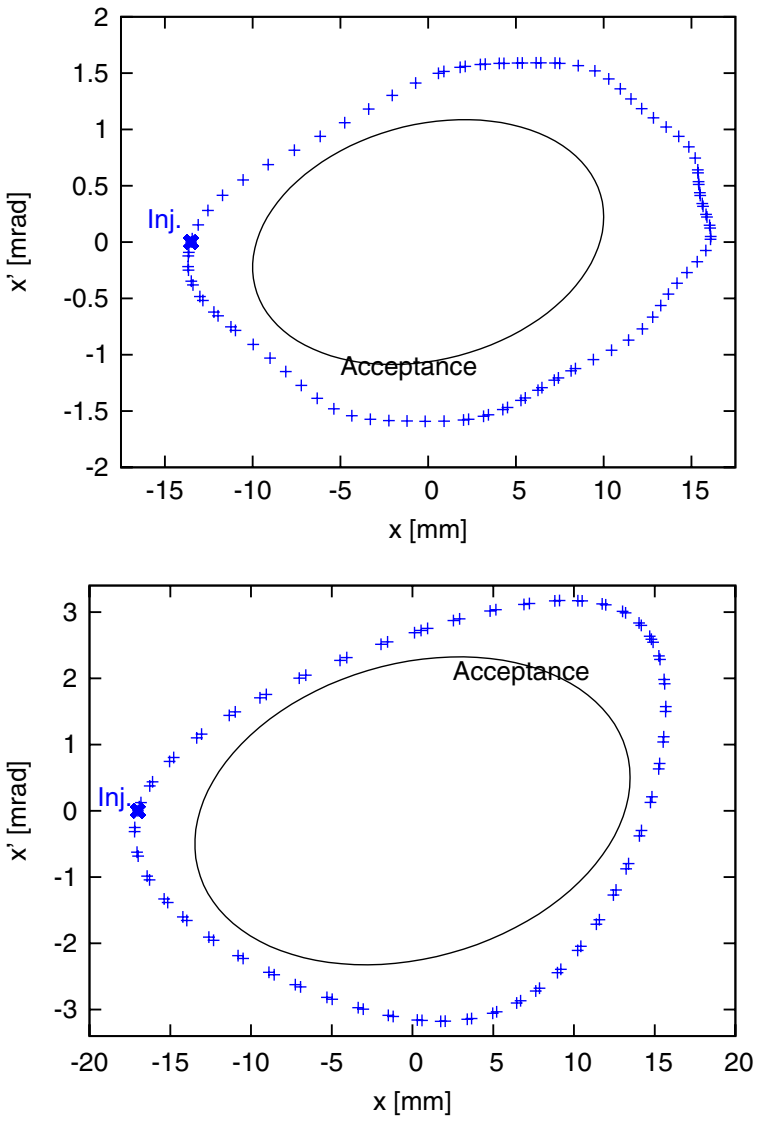

FIG. 9. Nonlinearity of betatron motion at large amplitudes. Phase space trajectory of the injected bunch at the end of the septum for 100 turns if no acceptance limitations were present in the MAX IV $3 \mathrm{GeV}$ storage ring (top) and $1.5 \mathrm{GeV}$ storage ring (bottom). 


$$
\left(b_{3} L\right)=\frac{\theta_{\mathrm{pm}}}{x_{\mathrm{pm}}^{2}} .
$$

The solutions for both MAX IV storage rings derived from tracking will be presented in the following sections.

\section{PULSED SEXTUPOLE INJECTION IN THE 3 GeV STORAGE RING}

The IP in the $3 \mathrm{GeV}$ storage ring is the magnetic end of the $5^{\circ}$ injection septum $(1902 \mathrm{~mm}$ downstream of the center of the injection straight or $444 \mathrm{~mm}$ upstream of the center of the beam position monitor (BPM) at the beginning of the first achromat). The optics in the horizontal plane at this location are $\beta_{\text {inj }}=9.402 \mathrm{~m}$ and $\alpha_{\text {inj }}=$ -0.211 . The lateral placement of the septum was chosen so that the blade becomes the limiting horizontal aperture of the storage ring $A_{x}^{2}=11.11 \mathrm{~mm}$ mrad while not limiting acceptance more than necessary. The injected bunch at the IP is at $x_{\text {inj }}=-13.5 \mathrm{~mm}$ (cf. Fig. 10) which gives an injected invariant of $A_{\mathrm{inj}}^{2}=20.25 \mathrm{~mm}$ mrad.

In the original injection scheme using a conventional four-kicker local bump, the separation between the injected bunch and the bumped stored beam was $5.5 \mathrm{~mm}$ which corresponded to a reduced invariant of $A_{\text {red }}^{2}=$ $3.36 \mathrm{~mm}$ mrad. This was taken as a target value for the reduced invariant using the PSM. A suitable location was found in the second long straight (cf. Figs. 11 and 12). At the beginning of this straight the amplitude of the injected bunch is too close to the acceptance but further into the straight the amplitude reduces somewhat. In order to keep the straight available to users, it was decided to put the PSM at the very end of this straight. There the amplitude of the injected bunch is still sufficiently large which keeps

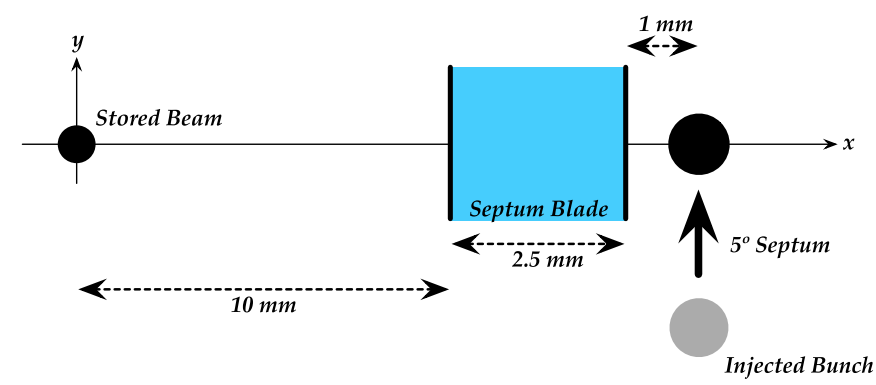

FIG. 10. Schematic of the situation at the IP in the MAX IV $3 \mathrm{GeV}$ storage ring looking downstream.
PSM strength manageable. It was decided to put the center of the PSM at the exact same location in the second long straight as the IP in the injection straight, and therefore the optics at the PSM are the same as at the IP.

At the PSM the coordinates of the injected bunch are $x_{\mathrm{pm}}=-4.665 \mathrm{~mm}$ and $x_{\mathrm{pm}}^{\prime}=1.067 \mathrm{mrad}$. Tracking reveals that reducing the divergence of the injected bunch at the PSM by $x_{\mathrm{pm}}^{\prime}=1.067 \rightarrow-0.1 \mathrm{mrad}$, i.e. $\theta_{\mathrm{pm}}=$ $1.167 \mathrm{mrad}$, minimizes the resulting reduced invariant at $A_{\text {red }}^{2}=2.31 \mathrm{~mm}$ mrad. The phase space plot in Fig. 13 (bottom) demonstrates clearly why the minimum invariant is reached with this kick. It should be noted that the reduced invariant achieved in this way is not only well within the storage ring acceptance $A_{x}$, but it also lies below what was achieved with the original injection scheme using the local four-kicker bump. The required PSM kick strength is given by Eq. (4) and using the above coordinates is calculated to be $\left(b_{3} L\right)=53.625 \mathrm{~m}^{-2}$.

\section{A. Injection efficiency}

So far only the ideal on-axis injected particle has been considered. In order to assess injection efficiency, the entire capture process of an actual injected bunch needs to be modeled. For this purpose the linac emittance and energy spread as well as the optics throughout the transfer have to be taken into account. As pointed out in the Introduction, the transfer lines mirror the optics at the linac

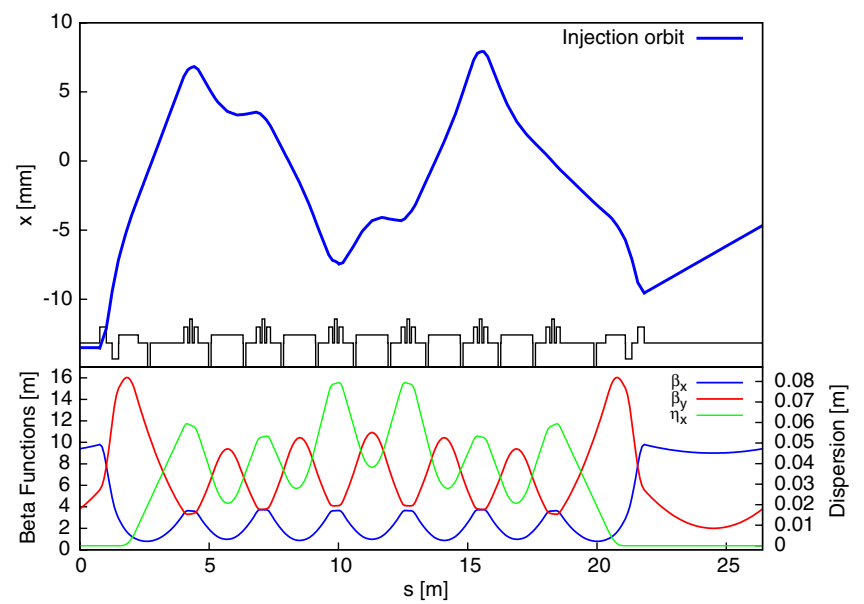

FIG. 12. Amplitude of the injected bunch (top) and optics (bottom) in the MAX IV $3 \mathrm{GeV}$ storage ring from the IP through the first achromat up to the PSM.

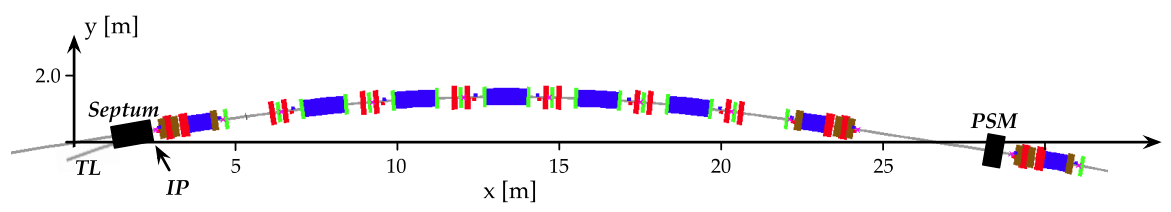

FIG. 11. Injection with the PSM into the MAX IV $3 \mathrm{GeV}$ storage ring. The IP at the end of the septum and the transfer line (TL) from the linac are indicated in the first straight. The PSM is located at the end of the second long straight. 

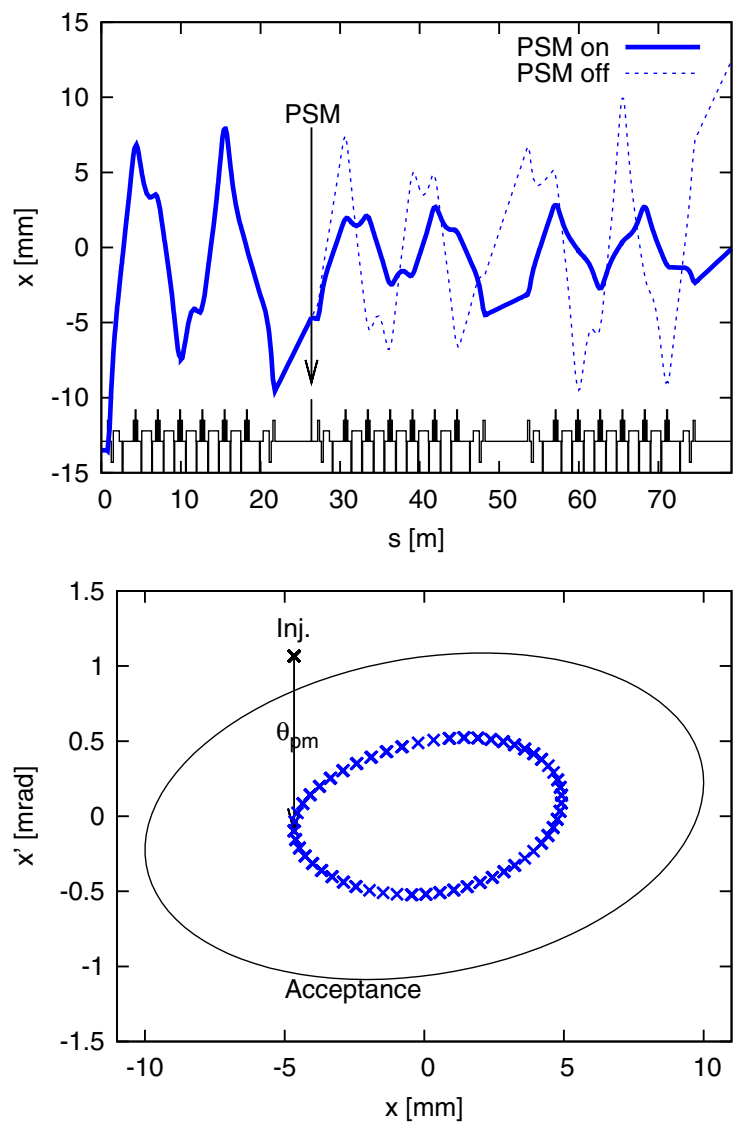

FIG. 13. Results of TRACY-3 tracking for injection and capture with the PSM in the $3 \mathrm{GeV}$ storage ring. Top: Amplitude of the injected bunch from the IP (end of the septum) through the first three achromats. Note that large amplitudes are only encountered in the achromat upstream of the PSM. Bottom: Phase space trajectory of the injected bunch at the location of the PSM for injection, capture, and the first 100 turns.

EP to the storage ring IP. Hence, the ensemble at the IP is defined by the linac extraction optics, linac emittance, and linac energy spread. This ensemble (i.e. the injected bunch) is then tracked through the storage ring as it receives a kick from the PSM and proceeds to orbit the machine for several turns. Ideally no particle loss is observed during this process.

An example for this procedure is displayed in Fig. 14 where injection and capture in the MAX IV $3 \mathrm{GeV}$ storage ring are shown for a bunch using a PSM in single-turn mode with the kick strength $\left(b_{3} L\right)$ calculated above. For the study a Gaussian particle distribution $(n=1000$, cutoff at $3 \sigma$ ) was tracked for five turns with DIMAD. The plot shows the data at the IP. The position of the septum is indicated. The normalized emittance of the linac is assumed to be $\varepsilon_{n}=10 \mathrm{~mm}$ mrad (corresponding to $\varepsilon_{x}=$ $1.7 \mathrm{~nm} \mathrm{rad}$ ), the energy spread is $\sigma_{\delta}=0.1 \%$. The transfer line optics are adjusted to mirror the linac EP optics to the storage ring IP according to the preferred linac optics mode [20]: $\beta_{x}=13.286 \mathrm{~m}$ (ideal IP optics would call for

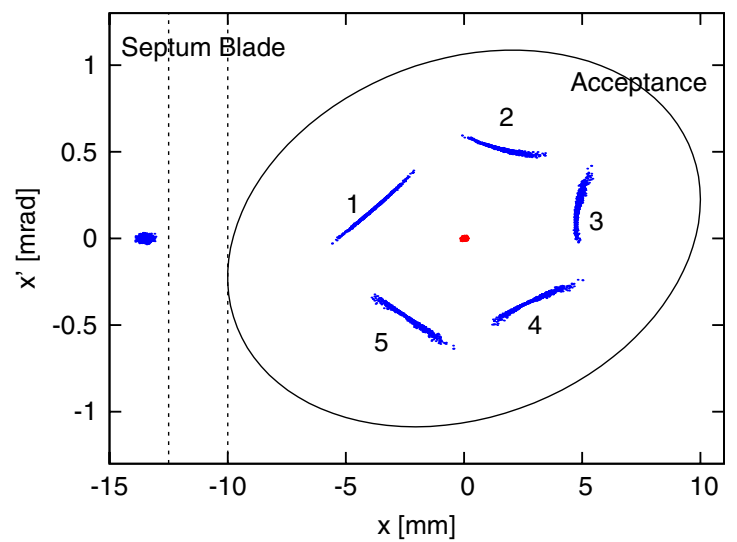

FIG. 14. DIMAD tracking data for injection, capture, and the first five turns in the storage ring shown at the IP. The injected bunch is modeled with a Gaussian particle distribution using 1000 particles with $\varepsilon_{n}=10 \mathrm{~mm} \mathrm{mrad}, \sigma_{\delta}=0.1 \%$, and a cutoff at $3 \sigma$ (blue). For comparison, the stored beam has been included (red).

$9.402 \mathrm{~m}$ ), $\alpha_{x}=-0.002$ (compared to -0.211 of the ideal IP optics). In this process no particles are lost which indicates injection efficiency should be very high.

As can be recognized by comparing the injected beam's size/shape before capture and after the first turn in Fig. 14, particle motion is subjected to considerable nonlinearity during capture. A priori, it is not clear if this is the result of the nonlinearity of the storage ring optics or the positiondependent kick received by the PSM. According to Eq. (4), the latter can be estimated as $\sigma_{\theta} / \theta_{\mathrm{pm}}=2 \sigma_{x} / x_{\mathrm{pm}} \approx 6 \%$. To illustrate, Fig. 15 shows the same situation, but replacing the nonlinear PSM kick with a pure dipole kick using the same 1.167 mrad nominal kick strength. By comparison, it can be concluded that the elongation of the bunch in transverse phase space after capture is primarily the result of the position-dependent PSM kick. As suggested by the above estimate, this emittance blowup is however small

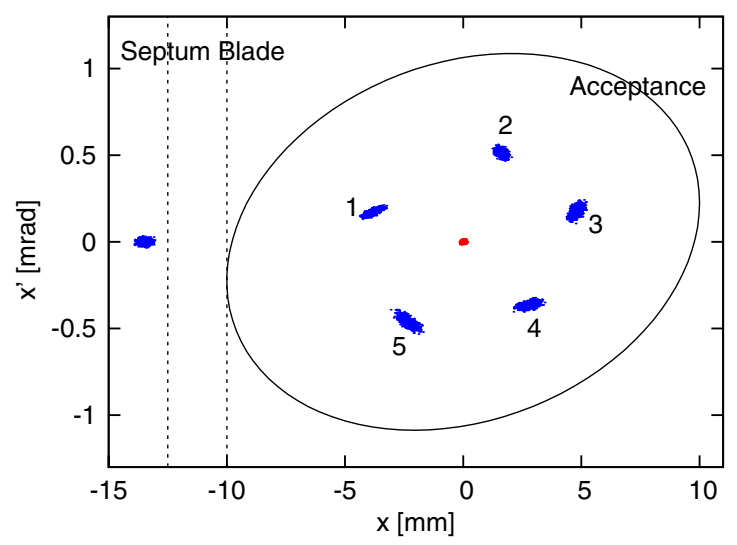

FIG. 15. The same situation as depicted in Fig. 14, but using a pure dipole kick instead of the sextupole kick. A comparison reveals the effect of the position dependence of the PSM kick. 
compared to the apparent emittance increase governed by the large betatron amplitudes of the injected bunch. Finally, the effect of the PSM on the stored beam is negligible, as already indicated in Fig. 8 (bottom).

It is important to point out here that, because of the lowemittance injection from the linac and the very large acceptance of the storage ring, a more elaborate matching of the transfer line optics to the storage ring optics at the IP (e.g. $[14,21,22])$ is not necessary to achieve high capture efficiency. In fact, the linac optics have been optimized for SPF operation and are not modified for ring injection shots. Obviously in reality, the linac could operate at slightly different optics or the extracted bunch could suffer an emittance and/or energy spread increase. In order to assess tolerances for linac operation, two extreme cases are displayed in Fig. 16 where capture efficiency is no longer $100 \%$. A situation where the energy spread of the injected bunch has increased eightfold to $\sigma_{\delta}=0.8 \%$ (which is actually already beyond the energy acceptance of the transfer line) shows smearing out of the injected bunch and tails starting to scrape the acceptance. The case with a sixfold increased emittance of $\varepsilon_{n}=60 \mathrm{~mm}$ mrad simulates emittance blowup in the linac as well as optics mismatches up
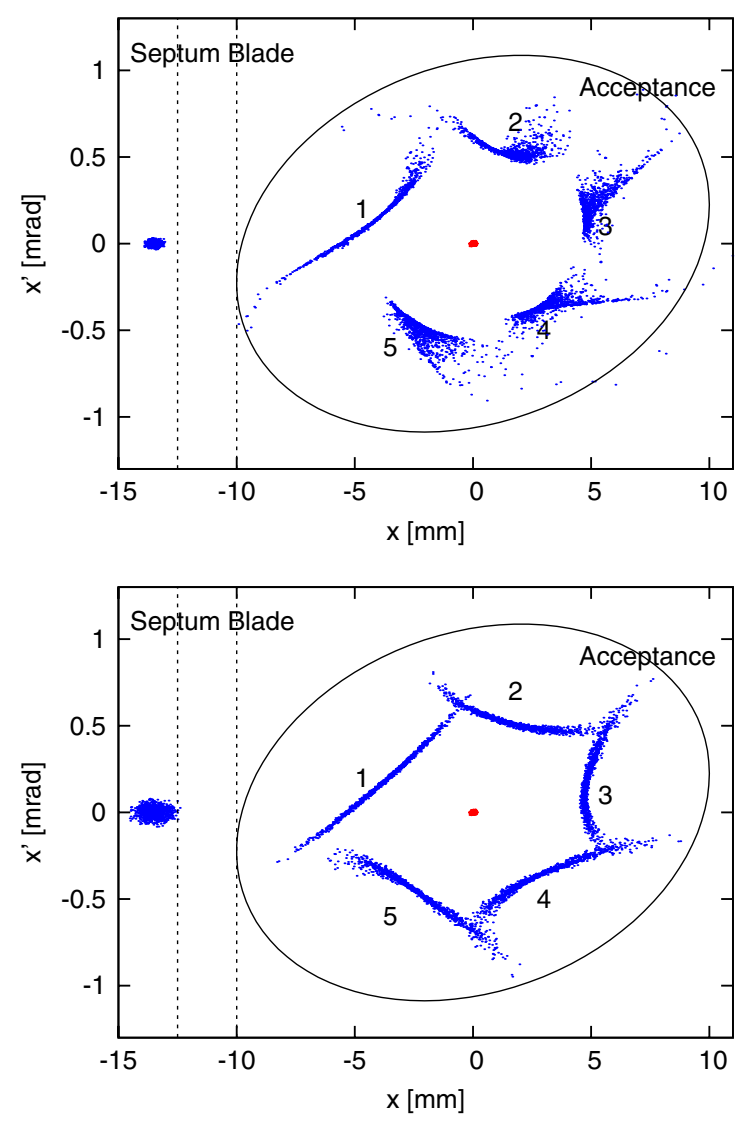

FIG. 16. DIMAD tracking data for mismatched injection. All parameters are identical to those used in Fig. 14 with the exception of an increased energy spread $\sigma_{\delta}=0.8 \%$ (top) and an emittance blowup $\varepsilon_{n}=60 \mathrm{~mm} \mathrm{mrad}$ (bottom). to the IP. In this situation a distortion of the bunch is observed. Any further increase of energy spread will lead to particle loss on the septum blade and the storage ring acceptance. Section VID provides more detail on PSM tolerances.

\section{B. Reduced-kick option}

To estimate feasibility of the PSM, the PSM kick strength $\left(b_{3} L\right)=53.625 \mathrm{~m}^{-2}$ calculated above for optimum single-turn injection can be compared to the kick strength achieved by the PSM operated in the PF at KEK. The KEK PSM is operated at $\left(b_{3} L\right)=6.4 \mathrm{~m}^{-2}$ giving $12 \mathrm{mT} \mathrm{m}$ at $15 \mathrm{~mm}$ [15]. However, the KEK PSM bore is $66 \mathrm{~mm}$ while the PSM in the MAX IV $3 \mathrm{GeV}$ storage ring can have a $36 \mathrm{~mm}$ bore diameter. The PSM pole-tip field required here is $579 \mathrm{mT}$ while $194 \mathrm{mT}$ have been achieved at KEK. If such a pole-tip field is not feasible, the length of the PSM can be increased from the $300 \mathrm{~mm}$ chosen at KEK. Regardless of the choice of length, it is of advantage to reduce the PSM strength requirement.

It is important to note that for capture to take place, the PSM has to kick the injected beam within the acceptance of the storage ring, but not necessarily to the minimum reduced invariant as performed above (cf. Fig. 14). Increasing the reduced invariant relaxes the kick strength requirement. Capture efficiency can remain high if the injected beam emittance and energy spread are close to design and optics mismatches can be kept sufficiently low. Examples of reduced-kick strengths are shown in Fig. 17. The minimum required kick for acceptance in the storage ring is $\theta_{\mathrm{pm}}=0.5 \mathrm{mrad}$ corresponding to $\left(b_{3} L\right)=23 \mathrm{~m}^{-2}$. The intermediate kick $(0.79 \mathrm{mrad})$ solution with $\left(b_{3} L\right)=$ $36.3 \mathrm{~m}^{-2}$ provides a substantial reduction of required PSM strength while still clearing the acceptance sufficiently to allow for some margin regarding optics mismatches and

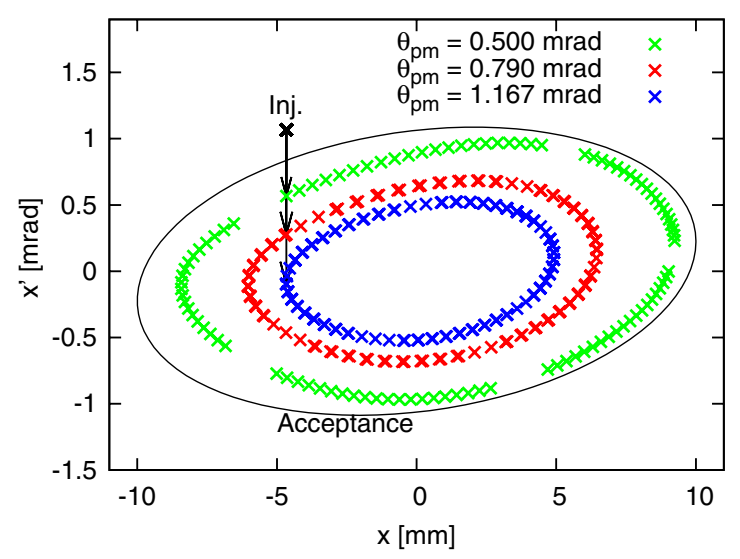

FIG. 17. Phase space trajectory of the injected bunch at the PSM in the MAX IV $3 \mathrm{GeV}$ storage ring as calculated with TRACY-3. Besides the design kick leading to a minimum reduced invariant (blue), two alternative injections with a reduced-kick strength are shown (red and green). 


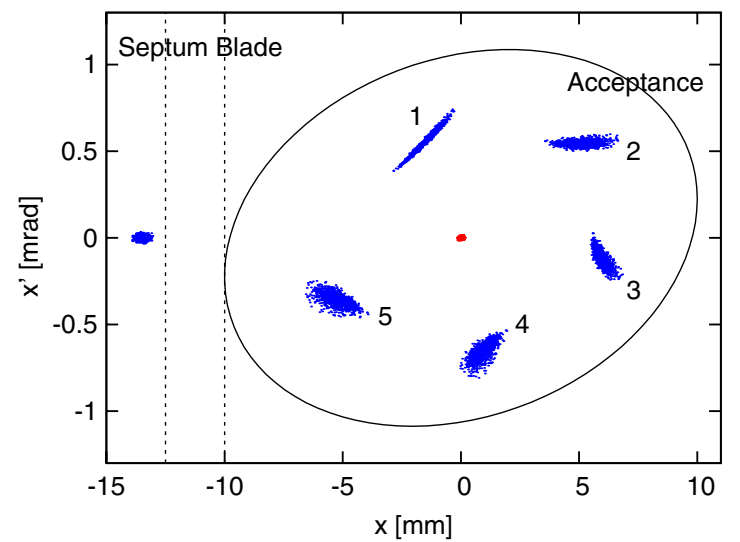

FIG. 18. Single-turn injection with reduced-kick strength $\left(b_{3} L\right)=36.3 \mathrm{~m}^{-2}$ corresponding to $\theta_{\mathrm{pm}}=0.79 \mathrm{mrad}$. DIMAD tracking data for injection, capture, and the first five turns in the storage ring shown at the IP. The injected bunch is modeled with a Gaussian particle distribution using 1000 particles with $\varepsilon_{n}=$ $10 \mathrm{~mm}$ mrad, $\sigma_{\delta}=0.1 \%$, and a cutoff at $3 \sigma$ (blue). For comparison, the stored beam has been included (red).

emittance and/or energy spread blowup of the injected bunch. The reduced invariant achieved in this way is $A_{\text {red }}^{2}=3.68 \mathrm{~mm} \mathrm{mrad}$. A phase space plot showing capture and the first five turns for this solution is displayed in Fig. 18. According to these results, capture efficiency can still be expected to be very high despite the reduced PSM kick strength.

\section{Two-turn injection option}

Another interesting possibility to reduce the required PSM strength while maintaining very high injection efficiency is multiturn injection. So far it has been tacitly assumed that the PSM kicks the injected bunch during one passage only. A simple pulser design would therefore deliver a half-sine pulse with base length no longer than two revolution periods. In multiturn injection, however, the PSM is excited by a pulse extending across more than two revolution periods. The injected bunch then receives a kick from the PSM kick on its first and subsequent passages. This not only reduces the required PSM strength, it also relaxes pulser requirements (crucial in small rings with short revolution periods, cf. Sec. V C). In principle, various pulse shapes are possible. For the considerations here a simple half-sine pulse shall be assumed. Also, because of the fractional horizontal tune of 0.20 only two-turn injection will be considered. For three-turn injection, the third kick would have the wrong sign.

In order to keep the injection scheme simple, a PSM with a half-sine pulse with base length $4 \times T_{\text {rev }}$ is assumed. The pulser is triggered so that the maximum amplitude $\left(b_{3} L\right)^{(0)}$ is reached when the injected bunch passes the PSM for the first time. When the injected bunch passes the PSM for the second time, it receives the reduced kick $\left(b_{3} L\right)^{(1)}=\sin (3 \pi / 4) \times\left(b_{3} L\right)^{(0)}$. On its third and subse- quent passages, the pulse amplitude is zero and the injected bunch will proceed to damp down to the stored beam. Given an injection invariant and a fixed PSM location, the kick strength to achieve a minimum reduced invariant after two turns can be calculated for linear betatron motion. In a real machine with nonlinear betatron motion, this minimum solution has to be derived from tracking. For the example presented here, a solution reasonably close to this minimum is investigated. The kick strength is roughly half of the kick strength used to minimize the reduced invariant in single-turn injection: $\left(b_{3} L\right)=28 \mathrm{~m}^{-2}$ (cf. Fig. 19). At the first passage $\left(b_{3} L\right)^{(0)}=28 \mathrm{~m}^{-2}$ generates a kick of -0.609 mrad reducing the invariant to $A_{\text {red }}^{(0) 2}=5.29 \mathrm{~mm}$ mrad. At the second passage the $\sqrt{2}$ reduction of field strength from the pulse shape along with the slightly larger amplitude of the injected bunch in the $\operatorname{PSM} x_{\mathrm{pm}}^{(1)}=4.784 \mathrm{~mm}$ combine to generate $\mathrm{a}-0.453 \mathrm{mrad}$ kick. A final reduced invariant of $A_{\text {red }}^{(1) 2}=2.99 \mathrm{~mm} \mathrm{mrad}$ is achieved. This is only $29 \%$ higher than the originally
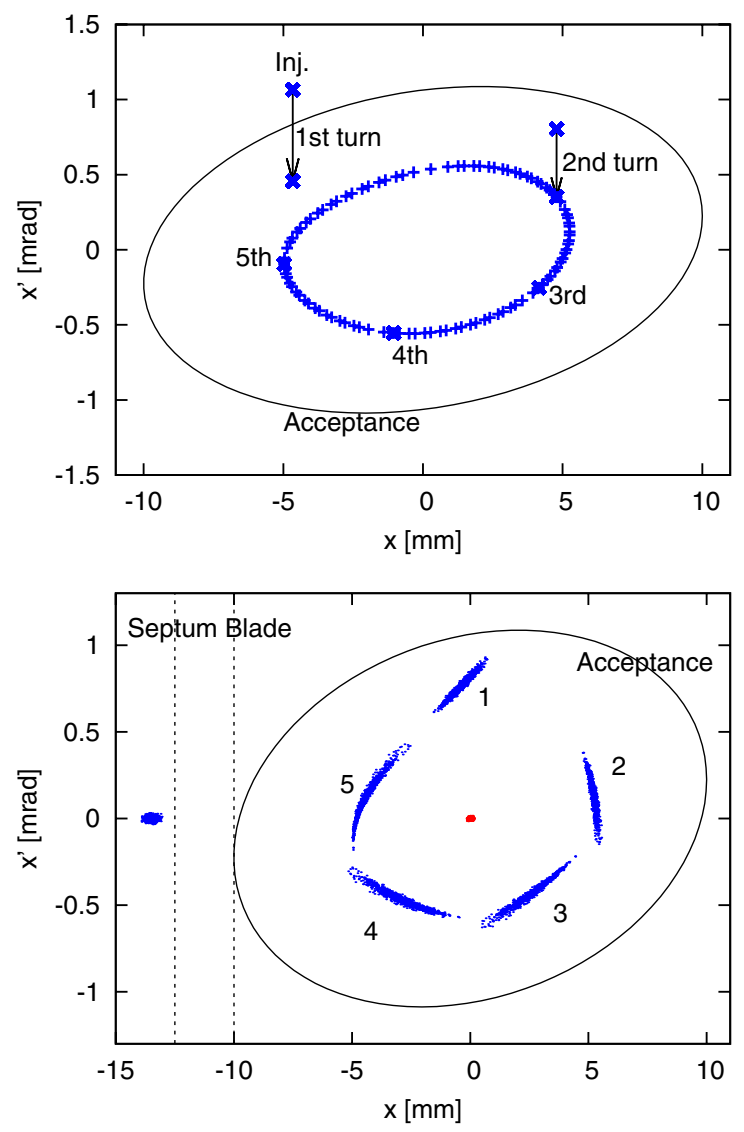

FIG. 19. Tracking results for capture of the injected bunch at the PSM using two-turn injection with $\left(b_{3} L\right)=28 \mathrm{~m}^{-2}$. Top: Phase space trajectory of the injected bunch at the PSM as calculated with TRACY-3. Bottom: DIMAD tracking data at the IP for injection and the first five turns in the storage ring. The injected bunch is modeled with a Gaussian particle distribution using 1000 particles with $\varepsilon_{n}=10 \mathrm{~mm} \mathrm{mrad}, \sigma_{\delta}=0.1 \%$, and a cutoff at $3 \sigma$. 
achieved reduced invariant, while the PSM strength requirement has been halved. Compared to the reduced-kick strength option presented in Sec. IV B, not only is the strength required here $23 \%$ lower, the resulting invariant is also $19 \%$ lower. Two-turn injection with reduced-kick strength shows the same negligible influence on the stored beam as single-turn injection with the PSM at nominal kick strength (cf. Fig. 8 bottom).

Two-turn injection offers potentially higher injection efficiency than single-turn injection with reduced-kick strength. On the other hand, it relies more heavily on the chosen optics. An adjustment of the (non)linear optics will change the position of the injected beam in the PSM. In single-turn injection the PSM can be retuned to achieve the proper kick, but in two-turn injection the kick strength cannot be adjusted individually for both passages. In such a situation, the maximum kick strength of the PSM can of course always be retuned to reduce the invariant after two kicks. Nevertheless, tuning freedom and the margin for error are narrower than in single-turn injection. Finally, it should be noted that the proposed two-turn injection scheme can only be realized with odd multipole magnets like the PSM as the kick in such magnets is symmetric in $x$.

\section{PULSED SEXTUPOLE INJECTION IN THE 1.5 GeV STORAGE RING}

As detailed in Sec. II, injection with a PSM is especially valuable in the $1.5 \mathrm{GeV}$ storage ring where only little space is available and a conventional injection bump would span several strong sextupoles and areas of large dispersion as well as encroach on two user straight sections. Analogous

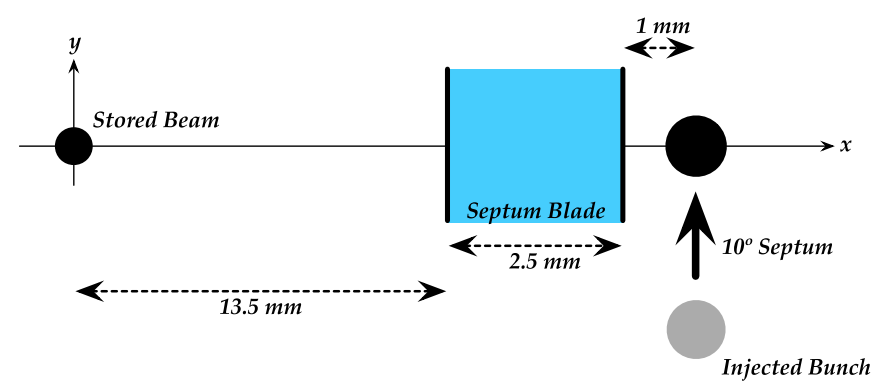

FIG. 20. Schematic of the situation at the IP in the MAX IV $1.5 \mathrm{GeV}$ storage ring looking downstream. to the $3 \mathrm{GeV}$ storage ring, the IP in the $1.5 \mathrm{GeV}$ storage ring is defined as the magnetic end of the $10^{\circ}$ injection septum (1252 mm downstream of the center of the injection straight). The optics in the horizontal plane at this location are $\beta_{\text {inj }}=5.960 \mathrm{~m}$ and $\alpha_{\text {inj }}=-0.220$. The lateral placement of the septum was again chosen so that the blade becomes the limiting horizontal aperture of the storage ring $A_{x}^{2}=32.06 \mathrm{mmmrad}$ while not limiting the lattice momentum acceptance (the limiting momentum aperture is at the center of the achromat where dispersion reaches the maximum value of $\eta_{x}=0.33 \mathrm{~m}$ ). The injected bunch at the IP is at $x_{\mathrm{inj}}=-17 \mathrm{~mm}$ (cf. Fig. 20) which gives an injected invariant of $A_{\mathrm{inj}}^{2}=$ $50.84 \mathrm{~mm}$ mrad.

A target value of $A_{\text {red }}^{2}=9 \mathrm{~mm}$ mrad was set for the reduced invariant using a PSM. This corresponds to a conventional injection using a four-kicker local bump with roughly $10 \mathrm{~mm}$ bump amplitude. Such a target cannot be met in the second straight section because of the large amplitude of the injected bunch (cf. Figs. 21 and 22). However, the beginning of the third straight section offers an excellent position for the PSM to reach the target. At the beginning of this straight, the amplitude of the injected bunch is large enough to limit PSM strength to achievable levels while small enough to match the reduced invariant target. A disadvantage of this choice is that the injected

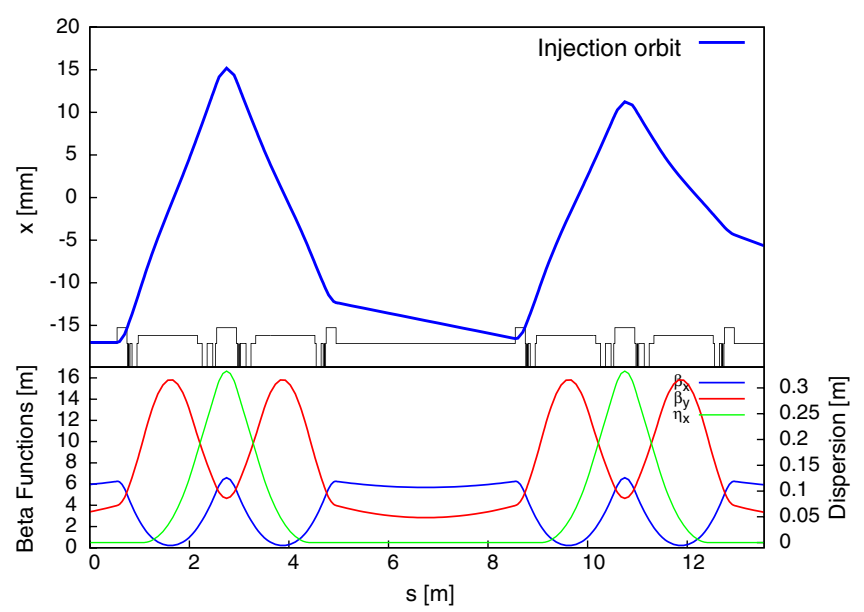

FIG. 22. Amplitude of the injected bunch (top) and optics (bottom) in the MAX IV $1.5 \mathrm{GeV}$ storage ring from the IP through the first two achromats up to the PSM.

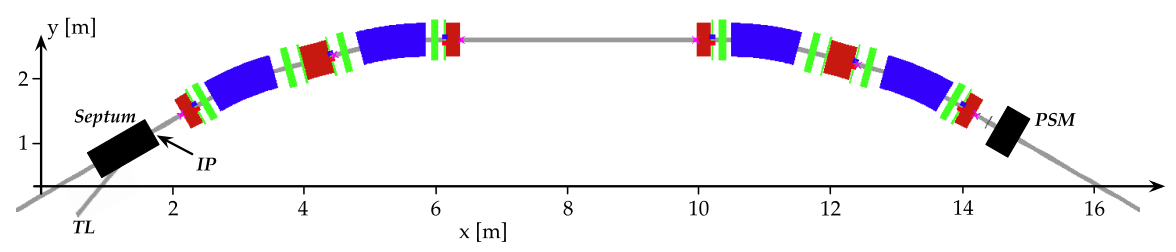

FIG. 21. Injection with the PSM into the MAX IV 1.5 GeV storage ring. The IP at the end of the septum and the transfer line (TL) from the linac are indicated in the first straight. The PSM is located at the beginning of the third straight. 
beam has to pass the first two achromats before reaching the PSM. Close attention to alignment and magnet strengths in these two achromats needs to be paid to ensure successful commissioning of the injection system.

The exact position of the center of the PSM is in the third straight, $1202 \mathrm{~mm}$ upstream of the center of the straight. At the location of the PSM the storage ring optics in the horizontal plane are $\beta_{\mathrm{pm}}=5.939 \mathrm{~m}$ and $\alpha_{\mathrm{pm}}=0.212$ and the coordinates of the injected bunch are $x_{\mathrm{pm}}=$ $-5.65 \mathrm{~mm}$ and $x_{\mathrm{pm}}^{\prime}=-2.16 \mathrm{mrad}$. Tracking reveals that kicking the injected bunch at the PSM by $x_{\mathrm{pm}}^{\prime}=-2.16 \rightarrow$ $+0.20 \mathrm{mrad}$, i.e. $\theta_{\mathrm{pm}}=2.36 \mathrm{mrad}$, minimizes the resulting reduced invariant at $A_{\text {red }}^{2}=5.38 \mathrm{~mm} \mathrm{mrad} \mathrm{(cf.} \mathrm{Fig.} \mathrm{23).}$ It should be noted that the reduced invariant achieved in this way is not only well within the storage ring acceptance $A_{x}$, but it also lies below the target, i.e., what can be achieved using a local four-kicker bump. The required PSM kick strength is given by Eq. (4) and using the above coordinates is calculated to be $\left(b_{3} L\right)=73.887 \mathrm{~m}^{-2}$.
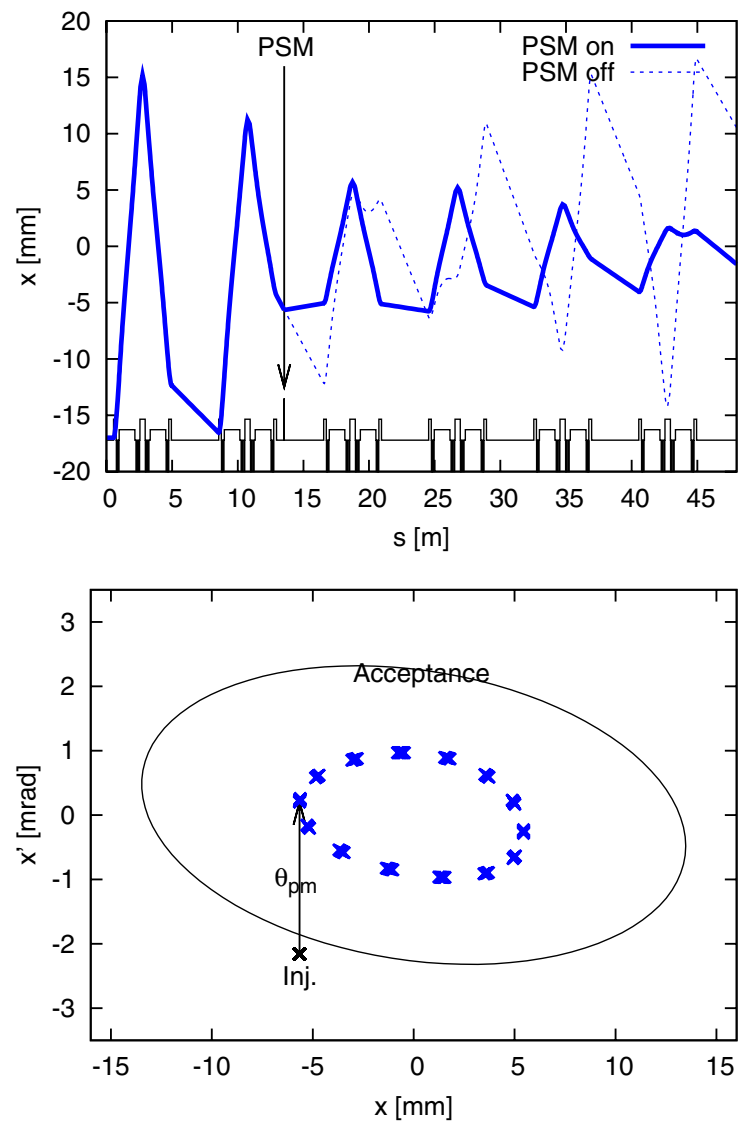

FIG. 23. Results of TRACY-3 tracking for injection and capture with the PSM in the $1.5 \mathrm{GeV}$ storage ring. Top: Amplitude of the injected bunch from the IP (end of the septum) through the first six achromats. Notice that large amplitudes are only encountered in the achromat upstream of the PSM. Bottom: Phase space trajectory of the injected bunch at the location of the PSM for injection, capture, and the first 100 turns.

\section{A. Injection efficiency}

Injection efficiency is again estimated by tracking an ensemble of particles representing the injected bunch. This is shown in Fig. 24 where injection and capture in the MAX IV $1.5 \mathrm{GeV}$ storage ring are shown for a bunch using a PSM in single-turn mode with the kick strength $\left(b_{3} L\right)$ calculated above. For the study, a Gaussian particle distribution ( $n=1000$, cutoff at $3 \sigma$ ) was tracked for five turns with DIMAD. The plot shows the data at the IP. The position of the septum is indicated. The normalized emittance of the linac is assumed to be $\varepsilon_{n}=10 \mathrm{~mm} \mathrm{mrad}$ (corresponding to $\varepsilon_{x}=3.4 \mathrm{~nm} \mathrm{rad}$ ), the energy spread is $\sigma_{\delta}=0.1 \%$. The transfer line optics are adjusted to mirror the linac EP optics to the storage ring IP according to the preferred linac optics mode [20]: $\beta_{x}=21.133 \mathrm{~m}$ (ideal IP optics would call for $5.960 \mathrm{~m}$ ), $\alpha_{x}=-0.002$ (compared to -0.220 of the ideal IP optics). In this process no particles are lost which indicates injection efficiency should be very high.

For comparison, Fig. 25 shows the same situation as Fig. 24, but instead of the nonlinear PSM kick, using a pure dipole kick with the same 2.36 mrad nominal kick strength. It can be concluded that although the position-dependent kick of the PSM causes some elongation of the bunch in transverse phase space after capture, this emittance blowup is small compared to the apparent emittance increase governed by the large betatron amplitudes of the injected bunch. Finally, as already seen in the $3 \mathrm{GeV}$ storage ring, the effect of the PSM on the stored beam in the $1.5 \mathrm{GeV}$ storage ring is negligible (cf. Fig. 26).

Limits for the amount of emittance and energy spread growth that can be tolerated can be inferred from Fig. 27. A situation where the energy spread of the injected bunch has increased to $\sigma_{\delta}=1.8 \%$ shows smearing out of the injected bunch and tails starting to scrape the acceptance

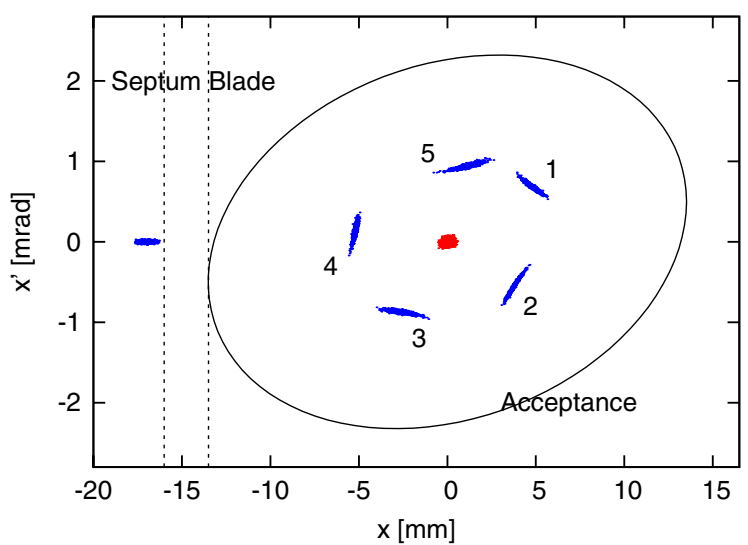

FIG. 24. DIMAD tracking data for injection, capture, and the first five turns in the storage ring shown at the IP. The injected bunch is modeled with a Gaussian particle distribution using 1000 particles with $\varepsilon_{n}=10 \mathrm{~mm} \mathrm{mrad}, \sigma_{\delta}=0.1 \%$, and a cutoff at $3 \sigma$ (blue). For comparison, the stored beam has been included (red). 


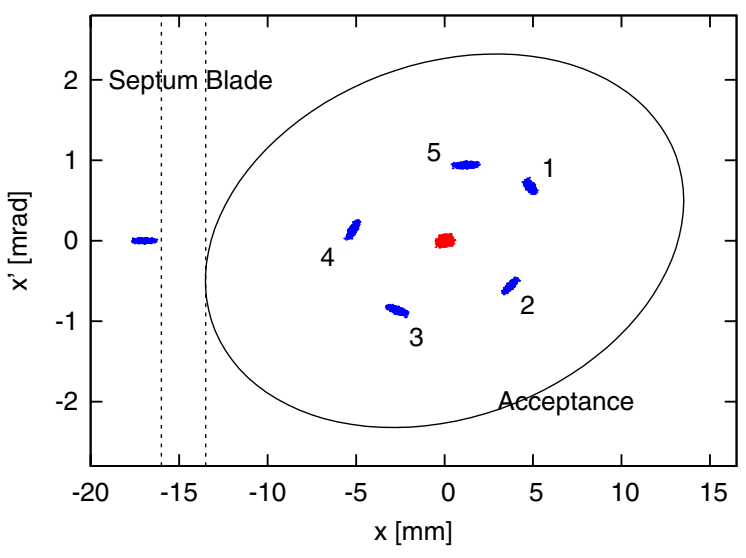

FIG. 25. The same situation as depicted in Fig. 24, but using a pure dipole kick instead of the sextupole kick. A comparison reveals the effect of the position dependence of the PSM kick.

(note however, that $1.8 \%$ is already beyond the transfer line's momentum acceptance). The case with a fourfold increased emittance of $\varepsilon_{n}=40 \mathrm{~mm}$ mrad simulates emittance blowup in the linac as well as optics mismatches up to the IP. In this situation no substantial distortion of the injected bunch is observed, however, the septum blade can no longer be cleared without particle loss at the nominal injection position of $x_{\text {inj }}=-17 \mathrm{~mm}$. Increasing the distance to the blade is possible if sufficient extra kick strength is available at the PSM. The large amount of emittance increase from the linac that can still be accepted by the $1.5 \mathrm{GeV}$ storage ring underlines the premise that a more elaborate matching of the injection optics to the storage ring is not required in order to reach high capture efficiency.

\section{B. Reduced-kick option}

The PSM strength $\left(b_{3} L\right)=73.887 \mathrm{~m}^{-2}$ derived above is high compared to the PSM used at the KEK PF. Despite

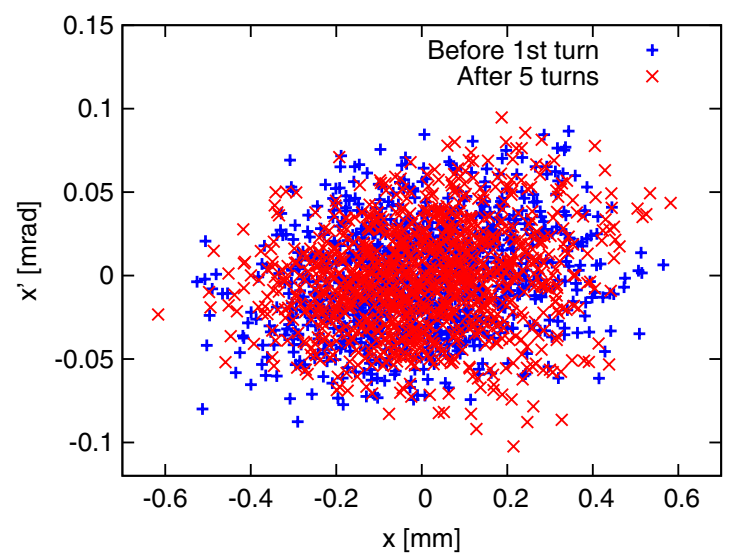

FIG. 26. DIMAD tracking results at the MAX IV $1.5 \mathrm{GeV}$ storage ring IP showing the effect of the pulsed magnet (single-turn injection mode) on the stored beam $\left(\varepsilon_{x}=\right.$ $6 \mathrm{~nm} \mathrm{rad}, \sigma_{\delta}=7.5 \times 10^{-4}, n=1000$, Gaussian distribution with cutoff at $3 \sigma$ ).
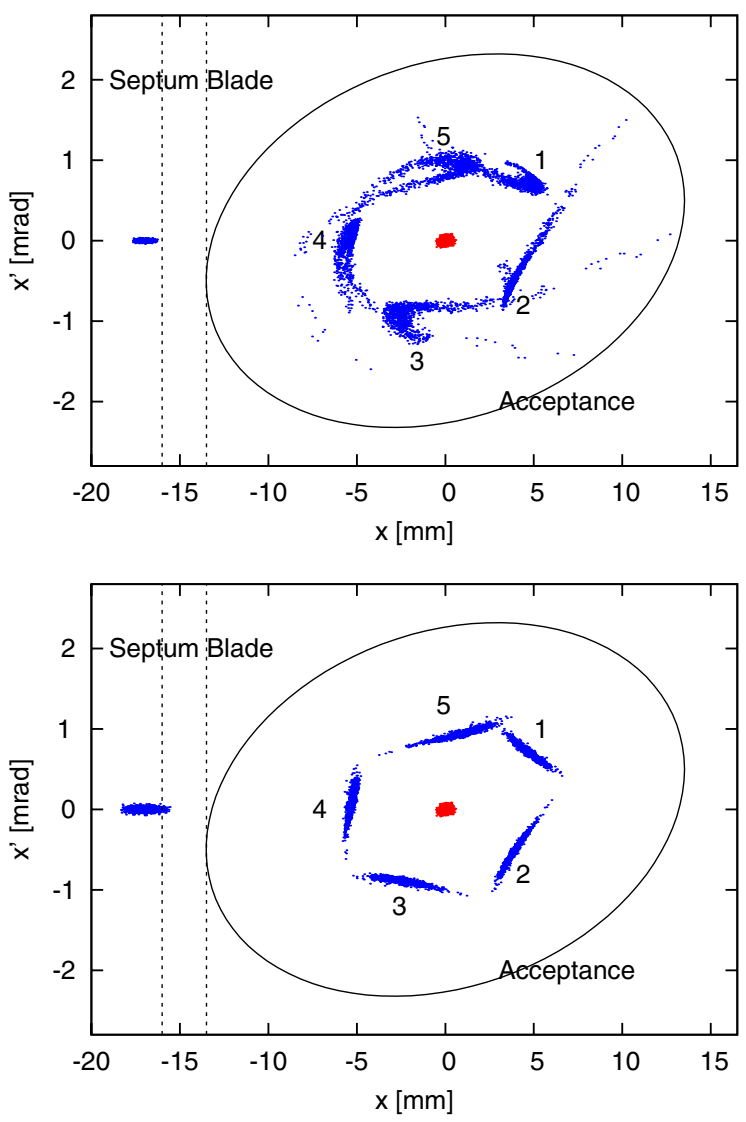

FIG. 27. DIMAD tracking data for mismatched injection. All parameters are identical to those used in Fig. 24 with the exception of an increased energy spread $\sigma_{\delta}=1.8 \%$ (top) and an emittance blowup $\varepsilon_{n}=40 \mathrm{~mm} \operatorname{mrad}$ (bottom).

the possibility to use a smaller magnet gap (44 $\mathrm{mm}$ full gap can be chosen for the PSM in the MAX IV $1.5 \mathrm{GeV}$ storage ring, cf. Sec. VI A) and a longer magnet length (400 mm can be used), the pole-tip field is $447 \mathrm{mT}$ which is roughly twice the value of the KEK PSM. It is again of interest to investigate alternate injection schemes where the PSM kick strength is reduced.

Considering the optics at the PSM (cf. Fig. 23, bottom) it is obvious that the PSM kick strength can be reduced without substantially increasing the reduced invariant. An example at the limit of what can be achieved in this way is shown in Fig. 28 where instead of the originally calculated PSM kick of $\theta_{\mathrm{pm}}=2.36 \mathrm{mrad}$, a reduced kick of $\theta_{\mathrm{pm}}=$ $0.85 \mathrm{mrad}$ corresponding to a PSM strength of $\left(b_{3} L\right)=$ $26.6 \mathrm{~m}^{-2}$ was applied. This reduces the required pole-tip field to $161 \mathrm{mT}$, well below what was achieved at KEK. The injected bunches are clearly still captured despite increasing the reduced invariant to $A_{\text {red }}^{2}=$ $18.98 \mathrm{~mm}$ mrad. Obviously, if the linac energy spread or emittance increases, or optics mismatches in the linac have to be accommodated for, the maximum possible reduction of kick strength is limited. 


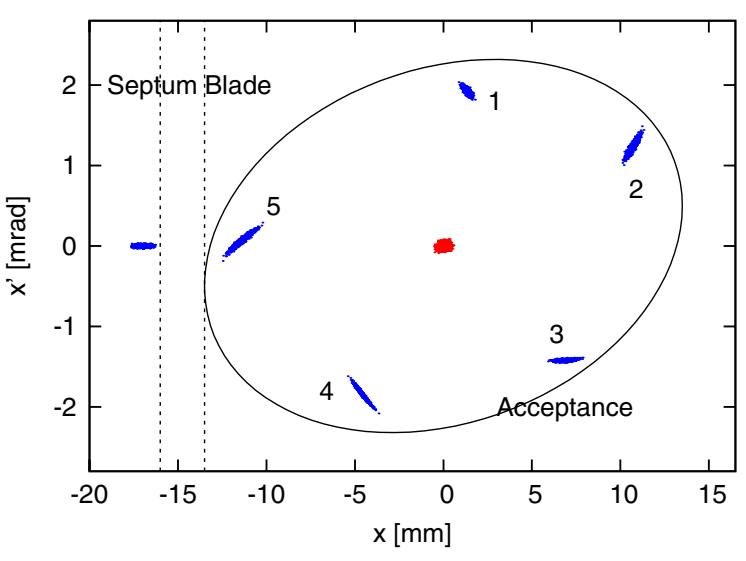

FIG. 28. Single-turn injection with reduced-kick strength $\left(b_{3} L\right)=26.6 \mathrm{~m}^{-2}$ corresponding to $\theta_{\mathrm{pm}}=0.85 \mathrm{mrad}$. DIMAD tracking data for injection, capture, and the first five turns in the storage ring shown at the IP. The injected bunch is modeled with a Gaussian particle distribution using 1000 particles with $\varepsilon_{n}=$ $10 \mathrm{~mm} \mathrm{mrad}, \sigma_{\delta}=0.1 \%$, and a cutoff at $3 \sigma$ (blue). For comparison, the stored beam has been included (red).

\section{Two-turn injection option}

A difficulty with PSM injection in the $1.5 \mathrm{GeV}$ storage ring is the pulse duration. Because of the ring's small circumference, the revolution period is only $0.32 \mu \mathrm{s}$. In order to facilitate pulser design, multiturn injection schemes have been investigated where the pulse duration can span more than two revolution periods. As in the case of the $3 \mathrm{GeV}$ storage ring, the $1.5 \mathrm{GeV}$ storage ring's fractional tune of 0.22 allows for no more than two-turn injection. Hence, such an injection scheme shall be presented here.

The required base length for the half-sine pulser excitation in two-turn injection for the $1.5 \mathrm{GeV}$ storage ring can be increased from $640 \mathrm{~ns}\left(2 \times T_{\text {rev }}\right)$ to $1.3 \mu \mathrm{s}\left(4 \times T_{\text {rev }}\right)$. Although this is still substantially lower than the requirement for the $3 \mathrm{GeV}$ storage ring injection pulser, it is considered feasible. Compared to the situation in the $3 \mathrm{GeV}$ storage ring, where both kicks reduce an injected particle's angle, the PSM operated in two-turn mode in the $1.5 \mathrm{GeV}$ storage ring can only reduce the injected particle's angle on its first passage. On the second passage the PSM kick increases the particle's angle due to the horizontal tune and the location of the PSM (cf. Fig. 23, bottom). Note also that this cannot be alleviated by choosing an even multipole magnet since the betatron oscillations of the injected particles have the same sign on both the first and second passage of the PSM.

Despite the wrong sign of the kick on the second passage, a sufficiently low reduced invariant can still be achieved with a properly adjusted PSM: the amplitude of the injected bunch at the PSM is so small on the second passage that the effective kick becomes negligible. The minimum reduced invariant that can be achieved in this way requires a PSM strength roughly equal to the ideal kick strength calculated for single-turn injection. It is interesting to note that increasing the kick strength does in fact not decrease the final invariant after both kicks. Therefore, for the example presented here, the exact same PSM strength shall be used as the one derived in singleturn injection. Tracking results are displayed in Fig. 29. During the first passage $\left(b_{3} L\right)^{(0)}=73.887 \mathrm{~m}^{-2}$ generates a kick of $+2.362 \mathrm{mrad}$ reducing the invariant to $A_{\text {red }}^{(0) 2}=$ $5.38 \mathrm{~mm}$ mrad. At the second passage, the amplitude is $-0.81 \mathrm{~mm}$ which, together with the $\sqrt{2}$ reduction of kick strength from the pulse shape, results in an almost negligible kick of $+34 \mu \mathrm{rad}$ and an almost unchanged final reduced invariant of $A_{\text {red }}^{(1) 2}=5.34 \mathrm{~mm} \mathrm{mrad}$.

It is obvious that using this method, two-turn injection does not lead to substantially lower reduced invariants than what can be achieved in single-turn injection. This approach does however allow doubling of the injection pulse duration without substantially increasing the final reduced
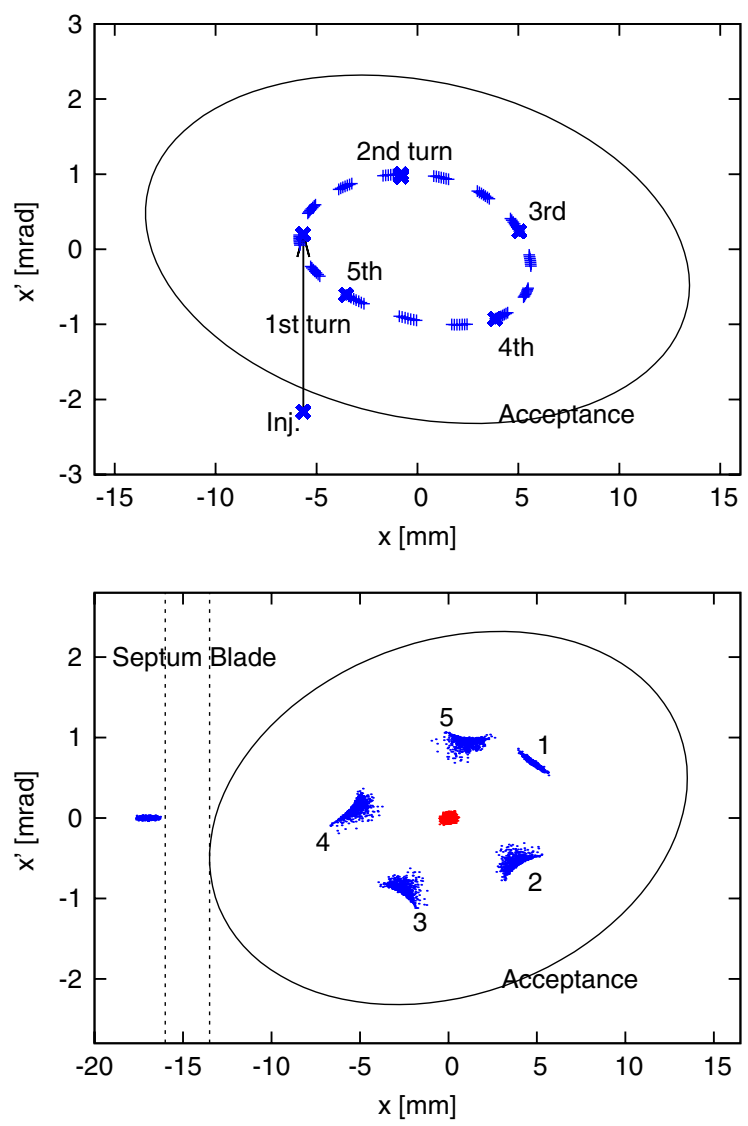

FIG. 29. Tracking results for capture of the injected bunch at the PSM using two-turn injection with $\left(b_{3} L\right)=73.89 \mathrm{~m}^{-2}$. Top: Phase space trajectory of the injected bunch at the PSM as calculated with TRACY-3 (the second kick can barely be seen because of the minute amplitude). Bottom: DIMAD tracking data at the IP for injection and the first five turns in the storage ring. The injected bunch is modeled with a Gaussian particle distribution using 1000 particles with $\varepsilon_{n}=10 \mathrm{~mm} \mathrm{mrad,} \sigma_{\delta}=0.1 \%$, and a cutoff at $3 \sigma$. 


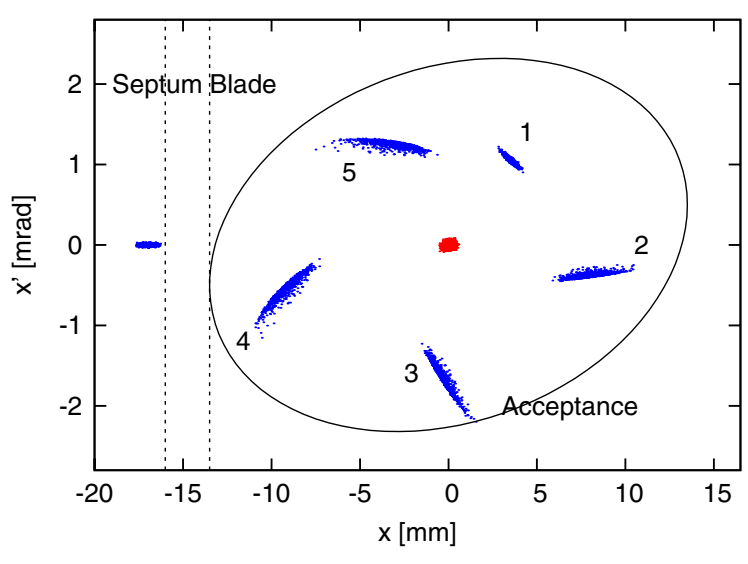

FIG. 30. Two-turn injection with reduced-kick strength $\left(b_{3} L\right)=59 \mathrm{~m}^{-2}$. DIMAD tracking data for injection, capture, and the first five turns in the storage ring shown at the IP. The injected bunch is modeled with a Gaussian particle distribution using 1000 particles with $\varepsilon_{n}=10 \mathrm{~mm} \mathrm{mrad}, \sigma_{\delta}=0.1 \%$, and a cutoff at $3 \sigma$ (blue). For comparison, the stored beam has been included (red).

invariant. In small rings with short revolution periods, this is an important improvement which allows one to relax otherwise exceedingly tight pulser requirements. Furthermore, two-turn injection as implemented here again shows the same negligible influence on the stored beam as single-turn injection with the PSM at nominal kick strength (cf. Fig. 26).

For the sake of completeness, a final example is presented where two-turn injection is performed using a reduced strength. The reduced strength derived in Sec. VB cannot be applied in two-turn mode for the reasons mentioned above. However, a reduced strength of $\left(b_{3} L\right)=$ $59 \mathrm{~m}^{-2}$ allows injection in two-turn mode without particle loss (cf. Fig. 30). This not only allows operating the PSM at a more relaxed pulse duration, but also $20 \%$ below nominal strength. The margin for error is obviously reduced in such a configuration. Nevertheless, it demonstrates the versatility of PSM injection into the $1.5 \mathrm{GeV}$ storage ring.

\section{TECHNICAL DESIGN AND TOLERANCES}

In order to procure the new pulsed magnet injection system, it was decided to generate an example PSM design which would serve potential suppliers as a reference. The following sections will briefly present this example design and the considerations behind.

\section{A. Magnet and vacuum chamber design}

A summary of the magnet specifications is displayed in Table I. Besides nominal values, it contains specifications for reduced-kick strength and two-turn injection.

An initial design of a PSM using ferrite yokes [23] is shown in Fig. 31. For the $3 \mathrm{GeV}$ storage ring PSM, a magnetic length of $300 \mathrm{~mm}$ and a bore diameter of
TABLE I. Pulsed sextupole magnet parameters for injection into the MAX IV storage rings.

\begin{tabular}{lccc}
\hline \hline & $B^{\prime \prime}\left[\mathrm{T} / \mathrm{m}^{2}\right]$ & $\mathrm{L}[\mathrm{m}]$ & $\tau[\mu \mathrm{s}]$ \\
\hline $3 \mathrm{GeV}$ PSM, nominal & 3575 & 0.3 & 3.5 \\
Reduced kick & 2420 & 0.3 & 3.5 \\
Two-turn injection & 1867 & 0.3 & 7.0 \\
\hline 1.5 GeV PSM, nominal & 1847 & 0.4 & 0.64 \\
Reduced kick & 665 & 0.4 & 0.64 \\
Two-turn injection & 1847 & 0.4 & 1.28 \\
Two-turn reduced-kick injection & 1475 & 0.4 & 1.28 \\
\hline \hline
\end{tabular}

$28 \mathrm{~mm}$ were chosen. This design foresaw a current of $1208 \mathrm{~A}$ to achieve the required kick in single-turn mode. However, closer inspection showed that the chosen magnet bore was too optimistic. The required beam-stay-clear full aperture at the $3 \mathrm{GeV}$ PSM is $22 \times 12 \mathrm{~mm}$. A ceramic chamber thickness of $5 \mathrm{~mm}$ and a gap between magnet and chamber of $2 \mathrm{~mm}$ for cooling should be considered. This results in a minimum magnet bore of $36 \times 26 \mathrm{~mm}$. For the $1.5 \mathrm{GeV}$ storage ring, the beam-stay-clear full aperture is $30 \times 11 \mathrm{~mm}$ which raises the minimum magnet bore to $44 \times 25 \mathrm{~mm}$. A redesigned PSM for this bore required a nominal excitation current of $1746 \mathrm{~A}$ and stored $14 \mathrm{~J}$ energy. The KEK PSM with its comparably large bore diameter of $66 \mathrm{~mm}$ stores $19.4 \mathrm{~J}$.

Because of the short pulse duration (especially in the $1.5 \mathrm{GeV}$ storage ring), the required voltage for the PSM can become rather large. Care therefore has to be taken to reduce the stored energy (for the above mentioned design a peak power of $67 \mathrm{MW}$ would be required in two-turn mode). One such attempt [24] was made by studying an asymmetric sextupole design where the poles were positioned according to the beam-stay-clear aperture. This reduced the stored energy and, hence, the required pulser power by about a factor of 3 . However, because of the nonlinear response of the ferrite, such an asymmetric sextupole design would not have zero field at the magnet center for all amplitudes (if an asymmetric octupole is used, the residual dipole is replaced with a residual quadrupole).

This problem can be eliminated by using iron instead of ferrite. Since the asymmetric design is very sensitive to coil placement errors, a recessed coil design where grooves are precision machined into the magnet yoke is of advantage. In such a design, a $100 \mu \mathrm{m}$ positioning error for the top coil gives rise to a $0.1 \mathrm{mT}$ dipole field at $0.4 \mathrm{~mm}$ from the center (roughly $3 \sigma_{x}$ of the injected beam in the $1.5 \mathrm{GeV}$ storage ring) which is considered acceptable (cf. Sec. VID). A further advantage of such an asymmetric design is that the field around the magnet center is flatter than in a symmetric sextupole. The example design using an asymmetric sextupole with an iron yoke for the $1.5 \mathrm{GeV}$ storage ring results in a stored energy of $7.4 \mathrm{~J}(L=$ $11.5 \mu \mathrm{H}, I=1134 \mathrm{~A})[25]$. In the $3 \mathrm{GeV}$ storage ring 


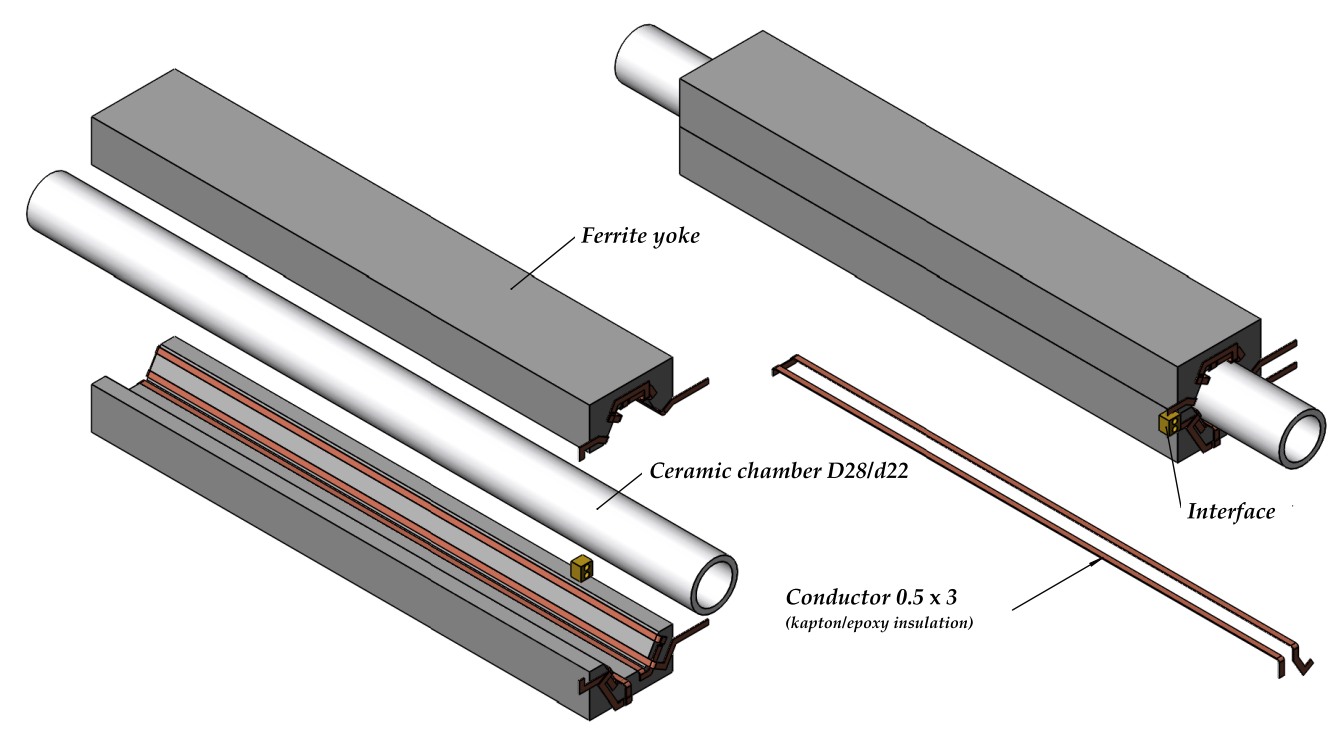

FIG. 31. Original reference design for a PSM for the MAX IV storage rings. The magnet consists of a ferrite yoke and 0.5 mm thick copper conductor surrounding a circular ceramic chamber with $3 \mathrm{~mm}$ chamber thickness and $22 \mathrm{~mm}$ inside diameter [23].

where beam stability tolerances are tighter (cf. Sec. VID), any residual field at the stored beam resulting from the asymmetric design is of concern. Therefore, the reference design for the $3 \mathrm{GeV}$ PSM foresees a symmetric sextupole despite the resulting increase of stored energy: $21 \mathrm{~J}(L=$ $9 \mu \mathrm{H}, I=2171 \mathrm{~A}$ ). With a symmetric iron sextupole, all pulse amplitudes should give zero field at the magnet center. Therefore no compensation coils (which usually entail a nontrivial design) to cancel dipole fields are required. On the other hand, exact alignment of the PSM to the stored beam is necessary in order to minimize dipole excitation of the stored beam. This will be discussed in more detail in Sec. VID.

A ceramic tube is chosen as a vacuum chamber to allow the quickly changing magnetic fields to penetrate the vacuum vessel without significant distortion from eddy currents. In order to prevent image charges from sensing a discontinuity in the chamber, the ceramic vessel will be coated internally with a thin conducting layer. Preliminary calculations show that a $5 \mu \mathrm{m}$ Ti coating restricts heating of the chamber to levels below $100 \mathrm{~W} / \mathrm{m}$ without deforming the pulse shape significantly or attenuating the field strength by more than $3 \%$.

In addition to the special ceramic chamber within the PSM, a modified section of the standard copper vacuum chamber for the $3 \mathrm{GeV}$ storage ring [1] needs to be provided where large amplitudes of the injected beam are expected. Apart from the injection straight where a widened chamber is foreseen, a special chamber section will also be installed at the last quadrupole of the first achromat where the injected beam approaches the standard chamber to within roughly $1 \mathrm{~mm}$ (cf. Fig. 12). For simplicity, it is being considered to use a mirrored copy of the same section in the injection straight. The extra $5 \mathrm{~mm}$ of horizontal aperture on the inside will provide an adequate safety margin for misalignments during commissioning. In the $1.5 \mathrm{GeV}$ storage ring, the amplitude of the injected beam is well within the $20-28 \mathrm{~mm}$ of horizontal chamber aperture foreseen for the standard vacuum chambers (cf. Fig. 22), hence no special chambers besides the actual PSM ceramic chamber are required for injection.

\section{B. Pulser design and timing}

The pulser requirements are derived from the presented PSM injection schemes. For single-turn injection the pulse shape is in principle not critical as long as fall time is short compared to the revolution period. Pulse length (for singleturn injection) should not exceed $640 \mathrm{~ns}$ and $3.5 \mu$ s for the 1.5 and $3 \mathrm{GeV}$ storage ring, respectively. Using these pulse lengths as well as the required current and stored energy given in Sec. VI A, the required voltages can be derived. The symmetric $3 \mathrm{GeV}$ PSM requires a pulser voltage of $17.5 \mathrm{kV}$ for single-turn injection (roughly the same voltage as achieved for the KEK PSM [15]), while the asymmetric design of the $1.5 \mathrm{GeV}$ storage ring operated in two-turn injection mode requires a voltage of $31.9 \mathrm{kV}$. The high voltage required by the $1.5 \mathrm{GeV}$ PSM is the result of the very short pulse duration.

Undershoot after the pulse should be limited, but is not of great concern. Peak-to-peak fluctuations of amplitude should be on the order of $\pm 0.1 \%$ and long-term drifts should be better than roughly $1 \%$. The PSM pulse is triggered by the global trigger system. Combined timing jitter of the PSM pulse with respect to the injected bunch should be better than \pm 5 ns over extended periods of time (typically one week). A typical example for the driving circuit of such a pulser is given in Fig. 16 in [11]. This example shows a thyratron-based pulser. If voltages permit, solid-state switches for the MAX IV injection pulsers 
TABLE II. Pulser requirements for PSM injection into the MAX IV $3 \mathrm{GeV}[1.5 \mathrm{GeV}]$ storage ring.

\begin{tabular}{lc}
\hline \hline Pulse length & $<3.5 \mu \mathrm{s}[640 \mathrm{~ns}]$ \\
Pulse length (two-turn injection) & $<7.0 \mu \mathrm{s}[1.28 \mu \mathrm{s}]$ \\
Fall time & $<1.8 \mu \mathrm{s}[320 \mathrm{~ns}]$ \\
Amplitude jitter within & $\pm 0.1 \%$ \\
Long-term amplitude drift & $<1 \%$ \\
Timing jitter within & $\pm 5 \mathrm{~ns}$ \\
Maximum repetition rate & $10 \mathrm{~Hz}$ \\
\hline \hline
\end{tabular}

can also be contemplated. A summary of pulser requirements is shown in Table II.

The jitter of the injected bunch train's arrival time with respect to the storage ring rf bucket is a crucial specification. Since this bunch train consists of three $3 \mathrm{GHz}$ bunches that are injected into a single $100 \mathrm{MHz}$ storage ring bucket, only one-third of the injected charge can arrive at the ideal phase with respect to the storage ring rf bucket. The other two-thirds are offset by \pm 330 ps. After a quarter synchrotron period (roughly 100 and 110 turns in the 1.5 and $3 \mathrm{GeV}$ storage ring, respectively), the \pm 330 ps phase error transforms into an energy offset of roughly $\delta= \pm 0.55 \%$ $(1.5 \mathrm{GeV})$ and $\pm 0.85 \%(3 \mathrm{GeV})$. Tracking studies have shown that injection energy acceptance in the $1.5 \mathrm{GeV}$ storage ring is significantly larger (cf. Fig. 27, top), however, in the $3 \mathrm{GeV}$ storage ring this approaches the limit of the energy acceptance during injection (cf. Fig. 16, top). Further tracking studies have therefore been performed to verify that the energy acceptance of the injection process does not limit capture efficiency for such a bunch train structure. A closer inspection reveals that injection energy acceptance for the $3 \mathrm{GeV}$ ring is larger below design energy and, in fact, capture efficiency is still very good at $\delta=-1.7 \%$. Therefore, injection with a phase offset of $330 \mathrm{ps}$ between the centroid of the injected bunches and the storage ring bucket (i.e. injecting the centroid at a phase corresponding to $\delta=-0.85 \%$ ) can be contemplated. This should guarantee high capture efficiency in the $3 \mathrm{GeV}$ storage ring for all three $3 \mathrm{GHz}$ bunches in the bunch trains coming from the linac.

The foreseen repetition rate is $10 \mathrm{~Hz}$ which is derived from the damping time in the storage rings. This results in five to ten damping times between injection shots in the $3 \mathrm{GeV}$ storage ring (depending on the installed insertion devices and gap settings) and more than ten damping times in the $1.5 \mathrm{GeV}$ storage ring. Special attention needs to be given to this figure in the case of Solaris [26] where this injection scheme will be duplicated, however for a ramped storage ring with injection at $550 \mathrm{MeV}$ [27]. At this low energy, the damping time is about $150 \mathrm{~ms}$ and an injected bunch will have only damped down to half its initial amplitude by the time the PSM pulser is triggered again. In the worst case this translates to roughly a quarter of the design kick being applied to the last injected bunch at the second pulse. Tracking studies show that this can blow up amplitudes by as much as $60 \%$ which is very close to the limit. If the injection energy cannot be raised to overcome this issue, a reduction of the repetition rate has to be contemplated.

Finally, it should be noted that possible pulser malfunction has no direct implications for machine protection or safety in the MAX IV storage rings. Injected bunches that do not receive the proper kick from the PSM will be lost on the vacuum chamber within the first turn. Because of the installation of a permanent magnet in each beam line front end [28], even grossly mis-steered bunches cannot be injected down a beam line. For top-up operation where frequent injection shots with open beam line shutters are foreseen, this is an important safety measure.

\section{Commissioning considerations}

During commissioning the proper trigger delay and pulser amplitude need to be determined. The MAX IV transfer lines and storage rings will be equipped with a state-of-the-art BPM system that can be operated in singleturn mode [1]. In both MAX IV storage rings, BPMs will be installed in the immediate vicinity of the PSM. The button sum signals from BPMs upstream of the PSM can be used to set the proper trigger delay.

The pulse amplitude will be adjusted so that the proper reduced orbit after the PSM is achieved. In single-turn mode the spacial resolution of the BPMs is limited, but better than the initial alignment accuracy of roughly $50 \mu \mathrm{m}$. Horizontal angular acceptance for injection at nominal PSM strength has been determined as $\pm 0.2 \mathrm{mrad}(1.5 \mathrm{GeV})$ and $\pm 0.1 \mathrm{mrad}(3.0 \mathrm{GeV})$ at the IP. This may appear rather limited, however, it should be compared to the angular acceptance of the injection septa. In these devices the injected bunches need to pass the narrow injection channel (at $1 \mathrm{~mm}$ separation from the septum blade) over a distance of $1 \mathrm{~m}$. The transfer lines contain sufficient BPMs and correctors so that position and angle can be determined and adjusted independently at the IP. In this way the spacial and angular acceptance of the injection channel can be scanned in order to achieve the design injection orbit. Fine-tuning of the transfer line dipoles and DC septa will finally be performed by verifying that bunches are injected at the IP at the design vertical position and angle.

Once beam can be accumulated in the storage rings, the spacial resolution of the BPMs will increase. Capture efficiency can then be optimized by adjusting the PSM pulse amplitude and timing with respect to the storage ring bucket while measuring the current in the storage rings. Especially in the $3 \mathrm{GeV}$ storage ring where many strong sextupoles and octupoles are installed between the IP and the PSM [16], a strong dependence of the injected orbit on the nonlinear optics has been observed. Considerable care needs to be taken to achieve the design injection orbit at the 
PSM to ensure that the PSM can generate a sufficiently strong kick at the amplitude of the injected bunch. If the PSM can deliver more kick strength than ideally required, this offers additional safety during early commissioning when substantial misalignments and erroneous nonlinear optics settings cannot be ruled out entirely.

A dipole kicker installed close to the IP is being contemplated as a backup measure to ensure that beam can be injected and stored during early commissioning even in the event of difficulties with the PSM. Such a dipole kicker can be installed close to a crossover of the injected beam so that nearly on-axis injection can be performed. Studies indicate the dipole kicker can be operated so that injected bunches can be kicked into the ring acceptance, while already captured bunches are not kicked out of the acceptance, i.e., accumulation becomes possible. Although this injection method is not compatible with top-up operation (unless the dipole kicker could be pulsed during the passage of only a single bunch, i.e., within $\leq 20 \mathrm{~ns}$ ), it allows storing beam for commissioning purposes (where tens of $\mathrm{mA}$ of current are required to reach maximum BPM resolution) as well as machine studies. Furthermore, such a dipole kicker can also serve as a horizontal pinger magnet in singlebunch operation.

\section{Tolerances}

In order to make top-up injection as transparent as possible to users, it is crucial to minimize the kick at the location of the stored beam in the PSM. This translates directly to an alignment tolerance for the PSM. Figure 32 shows the effect of a misaligned PSM on the stored beam of the $3 \mathrm{GeV}$ storage ring. The PSM has been misaligned by $50 \mu \mathrm{m}$ in the horizontal and the vertical planes. At nominal strength this corresponds to a $0.27 \mu \mathrm{rad}$ kick in the vertical (note that $\sigma_{y^{\prime}} \simeq 1 \mu \mathrm{rad}$ at the PSM).

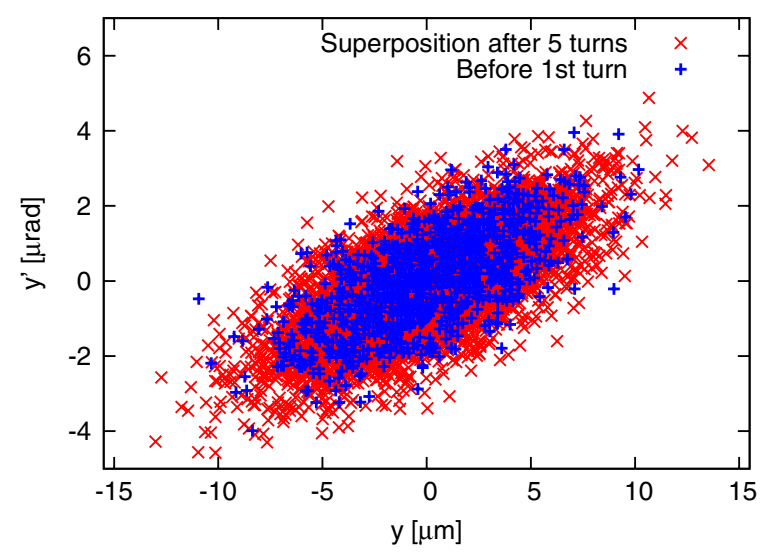

FIG. 32. DIMAD tracking results at the MAX IV $3 \mathrm{GeV}$ storage ring IP showing vertical perturbation of the stored beam $\left(\varepsilon_{x}=\right.$ $0.326 \mathrm{~nm} \mathrm{rad}, \kappa=1 \%, \sigma_{\delta}=7.7 \times 10^{-4}, n=1000$, Gaussian distribution with cutoff at $3 \sigma$ ) by a misaligned PSM in singleturn injection mode. The PSM has been misaligned by $50 \mu \mathrm{m}$ in the horizontal and the vertical planes.
TABLE III. Tolerances for misalignments and residual dipole fields on axis in the PSMs for the MAX IV storage rings.

\begin{tabular}{lc}
\hline \hline $3 \mathrm{GeV}$ PSM horizontal misalignment & $<96 \mu \mathrm{m}$ \\
$3 \mathrm{GeV}$ PSM vertical misalignment & $<10 \mu \mathrm{m}$ \\
$3 \mathrm{GeV}$ PSM integrated residual dipole field $(\mathrm{H})$ & $<1 \mu \mathrm{T} \mathrm{m}$ \\
$3 \mathrm{GeV}$ PSM integrated residual dipole field $(\mathrm{V})$ & $<5 \mu \mathrm{T} \mathrm{m}$ \\
$3 \mathrm{GeV}$ horizontal angular acceptance at IP & $\pm 0.1 \mathrm{mrad}$ \\
$1.5 \mathrm{GeV}$ PSM horizontal misalignment & $<202 \mu \mathrm{m}$ \\
$1.5 \mathrm{GeV}$ PSM vertical misalignment & $<10 \mu \mathrm{m}$ \\
$1.5 \mathrm{GeV}$ PSM integrated residual dipole field $(\mathrm{H})$ & $<1.5 \mu \mathrm{T} \mathrm{m}$ \\
$1.5 \mathrm{GeV}$ PSM integrated residual dipole field $(\mathrm{V})$ & $<15 \mu \mathrm{Tm}$ \\
$1.5 \mathrm{GeV}$ horizontal angular acceptance at IP & $\pm 0.2 \mathrm{mrad}$ \\
\hline \hline
\end{tabular}

As a target, perturbations to the stored beam shall be reduced to a level where any residual PSM kicks amount to less than $10 \%$ of the angular spread in the beam. For the $3 \mathrm{GeV}$ storage ring, this amounts to a maximum deflection angle of $0.5 \mu \mathrm{rad}$ in the horizontal and $0.1 \mu \mathrm{rad}$ in the vertical; correspondingly for the $1.5 \mathrm{GeV}$ storage ring, $3 \mu \mathrm{rad}$ in the horizontal and $0.3 \mu \mathrm{rad}$ in the vertical. At nominal PSM strength these kicks correspond to orbit offsets in the PSM of $\Delta x=96 \mu \mathrm{m}$ and $\Delta y=10 \mu \mathrm{m}$ in the $3 \mathrm{GeV}$ and $\Delta x=202 \mu \mathrm{m}$ and $\Delta y=10 \mu \mathrm{m}$ in the $1.5 \mathrm{GeV}$ storage ring. Such tight tolerances can only be met by careful alignment of the PSM with respect to the stored beam. To realign the PSM, horizontal and vertical bumps of varying amplitude can be driven through the PSM until perturbation of the stored beam is minimized. The PSM is then mechanically realigned by the amount indicated by the bump amplitudes. The supports of the PSM will be designed to facilitate this process.

Furthermore, even a perfectly aligned PSM can give rise to residual kicks to the stored beam. This can happen if, for example, the magnet symmetry is not perfect as a consequence of manufacturing errors. In such a situation, a beam passing the magnetic center of the PSM will probe a residual magnetic field. The amount of tolerable residual magnetic field at the magnetic center of the PSM can be derived from the limits for residual PSM kicks presented above. For the $3 \mathrm{GeV}$ storage ring, the maximum horizontal [vertical] magnetic field on axis is therefore $1 \mu \mathrm{Tm}$ [5 $\mu \mathrm{T} \mathrm{m}$ ]. For the $1.5 \mathrm{GeV}$ storage ring the maximum horizontal [vertical] magnetic field on axis is $1.5 \mu \mathrm{Tm}$ $[15 \mu \mathrm{T} \mathrm{m}]$. A summary of the alignment and residual field tolerances is given in Table III.

\section{SUMMARY AND OUTLOOK}

As the design of storage rings continues to improve to meet ever-increasing user requirements, not just lattice design and optics, but also technology needs to adapt. Special attention should be given to the injection process since conventional injection schemes were originally designed for medium to high emittance rings with limited acceptance, large magnets and apertures, as well as 
injection of high-emittance bunches. State-of-the-art storage rings have reduced the emittance to levels well below $1 \mathrm{~nm} \mathrm{rad}$ and rely on compact strong-focusing lattices using small magnets and narrow apertures. The injection scheme and the technology used for injection need to be adapted to better match these new rings. Pulsed multipole injection is such a novel approach.

An alternative injection scheme using pulsed sextupole magnets for the MAX IV storage rings has been presented. This injection scheme is simpler and takes up less space than conventional injection using a local four-kicker bump. Its main advantage however lies in the potential to make top-up injection transparent to beam line users despite the very high stability tolerances encountered in state-of-theart ultralow-emittance light sources. Hence, PSM injection will allow frequent low-charge top-up shots which will further improve stability in the storage ring. The proof of principle experiment for PSM injection has been carried out at the PF at KEK with great success [15]. It has therefore been decided that a conventional four-kicker bump will not be installed in the MAX IV storage rings at all. Instead, the MAX IV storage rings will be the first storage ring light sources designed to use PMI from the start. Expanding beyond the pioneering work performed at $\mathrm{KEK}$, this paper introduces multiturn injection schemes and quantitative analysis of injection efficiency and tolerances including phase shifts and jitter. The paper also demonstrates feasibility of PSM injection for an ultralow-emittance storage ring with its extremely tight tolerances on beam stability.

The presented injection schemes for the MAX IV storage rings are considered realistic and ready for procurement. In principle, a more aggressive approach could be pursued. By using an even thinner septum blade, bringing the septum closer to the stored beam, and injecting closer to the septum blade (or at an angle), a smaller reduced invariant can be achieved thus further increasing injection efficiency. It is important to note, however, that for PSM injection to remain feasible, the injected bunch needs sufficient amplitude in the PSM. Reducing the amplitude at injection can help to reduce the achievable reduced invariant, however, it usually also reduces the amplitude at the PSM thus requiring a stronger magnet. For a given PSM strength, a reduction of injection amplitude does not necessarily lead to a reduction of the reduced invariant.

As an alternative approach, a different multipole magnet for injection can be contemplated. As pointed out in Sec. III, the ideal multipole for injection would in principle be highly nonlinear, maximizing kick strength at the location of the injected beam while minimizing it in all other locations. If such a nonlinear kicker magnet can be designed with a flat field distribution around the center and possibly with a zero crossing (i.e. a position can always be found where the stored beam sees zero kick), it would offer further advantages over the PSM. Therefore, future injec- tion studies for the MAX IV storage rings will focus on the feasibility of such a nonlinear kicker magnet. Promising candidates have started to appear [17] and will hopefully be tested soon.

\section{ACKNOWLEDGMENTS}

The author would like to express his gratitude to Les Dallin (CLS) for many interesting discussions and example designs for pulsed sextupoles as well as his continued support. Thanks also go to Lars-Johan Lindgren (MAXlab) and Bengt Anderberg (AMACC) for producing example designs for the sextupole and pulser. Finally, the author would like to acknowledge Mikael Eriksson (MAX-lab) and Peter Kuske (HZB) for many stimulating exchanges on alternative injection ideas.

[1] MAX IV Detailed Design Report, available for download at http://www.maxlab.lu.se/maxlab/max4/index.html.

[2] M. Eriksson et al., in Proceedings of the 2011 Particle Accelerator Conference, NY, USA (IEEE, New York, 2011), TUOBS4.

[3] S. C. Leemann, A. Andersson, M. Eriksson, L.-J. Lindgren, E. Wallén, J. Bengtsson, and A. Streun, Phys. Rev. ST Accel. Beams 12, 120701 (2009).

[4] S. C. Leemann et al., in Proceedings of the IPAC'10 Conference, Kyoto, Japan (ICR, Kyoto, 2010), WEPEA058, p. 2618.

[5] S. Thorin et al., in Proceedings of the 2011 Particle Accelerator Conference, NY, USA (Ref. [2]), THP178.

[6] S. Werin, S. Thorin, M. Eriksson, and J. Larsson, Nucl. Instrum. Methods Phys. Res., Sect. A 601, 98 (2009).

[7] S. Werin (unpublished).

[8] L.-J. Lindgren and B. Anderberg, Nucl. Instrum. Methods Phys. Res., Sect. A 490, 592 (2002).

[9] L.-J. Lindgren and B. Anderberg, Nucl. Instrum. Methods Phys. Res., Sect. A 547, 686 (2005).

[10] P. Kuske, at the Top-Up Workshop, Melbourne, Australia, 2009.

[11] K. Harada, Y. Kobayashi, T. Miyajima, and S. Nagahashi, Phys. Rev. ST Accel. Beams 10, 123501 (2007).

[12] P. Kuske et al., in Proceedings of the 11th European Particle Accelerator Conference, Genoa, 2008 (EPS-AG, Genoa, Italy, 2008), WEPC037, p. 2067.

[13] T. Shaftan et al., in Proceedings of the 23rd Particle Accelerator Conference, Vancouver, Canada, 2009 (IEEE, Piscataway, NJ, 2009), TU5RFP012, p. 1114.

[14] C. Sun et al., in Proceedings of the IPAC'10 Conference, Kyoto, Japan (Ref. [4]), WEPEA068, p. 2642.

[15] H. Takaki, N. Nakamura, Y. Kobayashi, K. Harada, T. Miyajima, A. Ueda, S. Nagahashi, M. Shimada, T. Obina, and T. Honda, Phys. Rev. ST Accel. Beams 13, 020705 (2010).

[16] S. C. Leemann and A. Streun, Phys. Rev. ST Accel. Beams 14, 030701 (2011).

[17] T. Atkinson et al., in Proceedings of IPAC'11, San Sebastián, Spain, 2011, THPO024, p. 3394. 
[18] H. Takaki et al., in Proceedings of the 23rd Particle Accelerator Conference, Vancouver, Canada, 2009 (Ref. [13]), TU6RFP045, p. 1647.

[19] R. Takai et al., in Proceedings of the 10th European Workshop on Beam Diagnostics and Instrumentation for Particle Accelerators, Hamburg, Germany, 2011 (DESY, Hamburg, 2011), TUPD03.

[20] S. Thorin (unpublished).

[21] A. Streun, SLS Report No. SLS-TME-TA-20020193, 2002 [http://ados.web.psi.ch/slsnotes/tmeta020193 .pdf].
[22] R. P. Fliller, in Proceedings of the IPAC'10 Conference, Kyoto, Japan (Ref. [4]), TUPEC040, p. 1814.

[23] B. Anderberg (unpublished).

[24] L. Dallin (unpublished).

[25] L. Dallin (unpublished).

[26] C. Bocchetta et al., in Proceedings of IPAC'11, San Sebastían, Spain, 2011, THPC054, p. 3014.

[27] A. Wawrzyniak et al., in Proceedings of IPAC'11, San Sebastían, Spain, 2011, THPC123, p. 3173.

[28] Y. Cerenius and P. Sondhauss, Internal MAX-lab Report, version 2.2, 2011. 